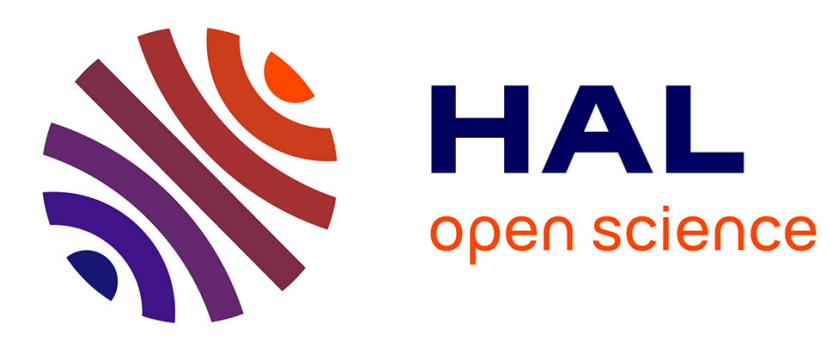

\title{
Finite element discretization of the time dependent axisymmetric Darcy problem
}

Christine Bernardi, Ajmia Younes Orfi

\section{To cite this version:}

Christine Bernardi, Ajmia Younes Orfi. Finite element discretization of the time dependent axisymmetric Darcy problem. 2014. hal-01073998

\section{HAL Id: hal-01073998 \\ https://hal.sorbonne-universite.fr/hal-01073998}

Submitted on 11 Oct 2014

HAL is a multi-disciplinary open access archive for the deposit and dissemination of scientific research documents, whether they are published or not. The documents may come from teaching and research institutions in France or abroad, or from public or private research centers.
L'archive ouverte pluridisciplinaire HAL, est destinée au dépôt et à la diffusion de documents scientifiques de niveau recherche, publiés ou non, émanant des établissements d'enseignement et de recherche français ou étrangers, des laboratoires publics ou privés. 


\title{
Finite element discretization of the time dependent axisymmetric Darcy problem
}

\author{
Christine Bernardi ${ }^{1}$ and Ajmia Younes Orfi ${ }^{2}$
}

\begin{abstract}
${ }^{1}$ Laboratoire Jacques-Louis Lions, C.N.R.S. Université Pierre et Marie Curie, B.C. 187, 4 Place Jussieu, 75252 Paris Cedex 05, France

CNRS, UMR 7598, Laboratoire Jacques-Louis Lions, F-75005, Paris, France Sorbonne Universits, UPMC Univ Paris 06, UMR 7598, Laboratoire JacquesLouis Lions, F-75005, Paris, France

e-mail: bernardi@ann.jussieu.fr

${ }^{2}$ University of Tunis El Manar, Faculty of Sciences, 1060 Tunis, Tunisie e-mail: ayounesorfi@yahoo.com

\section{Abstract}

We consider the Darcy problem in an axisymmetric three-dimensional domain with data which are axisymmetric. The solution satisfies a system of equations in the meridian domain. We propose a discretization of this problem in the case of an axisymmetric solution. This discretization relies on a backward Euler's scheme for the time variable and finite elements for the space variables. We prove a priori error estimates and a posteriori error estimates both for the time steps and the meshes and we present some numerical experiments which are in good agreement with the analysis.
\end{abstract}

Keywords: Darcy's equations; axisymmetric domain; time discretization; finite element discretization; a posteriori analysis.

\section{Introduction}

Let $\breve{\Omega}$ be a bounded three-dimensional domain which is invariant by rotation around an axis. The boundary $\breve{\Gamma}$ of this domain is divided into two parts $\breve{\Gamma}_{p}$ and $\breve{\Gamma}_{u}$. We are interested in the following model, suggested by Rajagobal [14],

$$
\begin{cases}\partial_{t} \breve{\boldsymbol{u}}+\alpha \breve{\boldsymbol{u}}+\operatorname{grad} \breve{p}=\breve{\boldsymbol{f}} & \text { in } \breve{\Omega} \times] 0, T[, \\ \operatorname{div} \breve{\boldsymbol{u}}=0 & \text { in } \breve{\Omega} \times] 0, T[, \\ \breve{p}=\breve{p}_{b} & \text { on } \left.\breve{\Gamma}_{p} \times\right] 0, T[, \\ \breve{\boldsymbol{u}} . \breve{\boldsymbol{n}}=\breve{g} & \text { on } \left.\breve{\Gamma}_{u} \times\right] 0, T[, \\ \breve{\boldsymbol{u}}=\breve{\boldsymbol{u}}_{0} & \text { in } \breve{\Omega} \text { at } t=0 .\end{cases}
$$

where the unknowns are the velocity $\breve{\boldsymbol{u}}$ and the pressure $\breve{p}$ of the fluid. The data are the quantities $\breve{f}, \breve{g}$, the pressure on the boundary $\breve{p}_{b}$ and the initial values of 
the velocity $\breve{\boldsymbol{u}}_{0}$. The parameter $\alpha$ is a positive constant representing the drag coefficient. If the problem is set in a domain which is symmetric by rotation around an axis, it is proved in [6] that, when using a Fourier expansion with respect to the angular variable, a three-dimensional problem is equivalent to a system of two-dimensional problems on the meridian domain, each problem being satisfied by Fourrier coefficient of the solution. Here we are going to present an axisymmetric model, and we propose a discretization of this problem in the case of an axisymmetric solution, i.e. only for the Fourier coefficient of order 0 .

We recall that the problem considered in [7] which is similar to the present problem is restricted to a boundary condition for the pressure and the case where the domain is a general two- or three-dimensional with a Lipschitz-continuous boundary. In the present study, mixed boundary conditions are considered, and we treat the problem in the simpler case of the meridian domain $\Omega$ where the data are axisymmetric, in the sense of $[6$, Sec. II.3]. So, by using cylindrical coordinates, we can write a variational formulation of this problem in the meridian domain. We prove the well-posedness and some regularity properties of an axisymmetric solution for such a system. Next, we propose a time semidiscrete problem that relies on the backward Euler's scheme. We prove that this problem has a unique solution and derive error estimates. Concerning the space discretization, we consider a conforming finite element method which leads to a well-posed discrete problem for which we prove a priori error estimates. We introduce two families of error indicators, one for the time semi-discretization and another one for the space discretization. We also prove a posteriori error estimates which are optimal according to the standard criteria, see [16]. Therefore the error indicators that we propose seem appropriate to perform time and space adaptivity in an efficient way. In a final step, we propose an algorithm for solving the resulting system and present some numerical experiments.

An outline of the paper is as follows:

- In Sectin 2, we write a variational formulation of problem (1) in the case of an axisymmetric solution, and we prove its well-posedness.

- Sectin 3 is devoted to the description and a priori analysis of the discrete problem in the meridian domain $\Omega$.

- In Sectin 4, two families of error indicators are proposed and the a posteriori analysis of the discrete problem is carried out.

- In Sectin 5, we present some numerical experiments.

\section{The two-dimensional formulation}

We are interested in modeling a flow through a bounded and symmetric domain $\breve{\Omega}$ with respect to the $z$ axis. We use cylindrical coordinates $(r, \theta, z)$. So the axisymmetric domain $\breve{\Omega}$ is the three-dimensional set obtained by rotating the two-dimentional domain $\Omega$, called meridian domain, around the axis $r=0$. The domain $\breve{\Omega}$ is defined as:

$$
\left.\left.\breve{\Omega}=\left\{(r, \theta, z) ;(r, z) \in \Omega \cup \Gamma_{0} \text { and } \theta \in\right]-\pi, \pi\right]\right\},
$$


where $\Gamma_{0}$ is the intersection of $\breve{\Omega}$ with the axis $r=0$. For simplicity, we assume that $\Gamma_{0}$ is the union of a finite number of segments with positive measure.

The fluid is modeled by the axisymmetric Darcy equations (1) in the domain $\breve{\Omega}$, we suppose that the boundary conditions and the external forces are axisymmetric and that their angular component is zero. So, we are interested with problem in the special case of an axisymmetric geometry and for axisymmetric data. The two-dimensional axisymmetric boundary $\breve{\Gamma}$ of the physical domain $\breve{\Omega}$ is a Lipschitz-continuous boundary and is divided into two parts $\breve{\Gamma}_{p}$ and $\breve{\Gamma}_{u}$, also with Lipschitz continous boundaries. The part of the boundary $\breve{\Gamma}_{p}$ has a positive surface measure. $\breve{\Gamma}_{u}=\breve{\Gamma} \backslash \bar{\Gamma}_{p}$ is the union of a finite number of surface elements.

Setting $\Gamma=\partial \Omega \backslash \Gamma_{0}$ and rotating $\Gamma$ around the axis $r=0$ gives back $\breve{\Gamma}$, and $\Gamma_{0}$ is a kind of artificial boundary. We also introduce the two parts $\Gamma_{p}$ and $\Gamma_{u}=\Gamma \backslash \bar{\Gamma}_{p}$ of the boundary of $\Gamma$. The unit outward normal vector $\breve{\boldsymbol{n}}$ on $\breve{\Gamma}$ is obtained by rotating the unit outward vector $\boldsymbol{n}$ on $\Gamma$. An axisymmetric function $\breve{p}$ on $\breve{\Omega}$ depends only on the radial and axial coordinates, therefore we associate a function $p$ on $\Omega$ such that $p(r, z)=\breve{p}(r, 0, z)$. An axisymmetric vector field $\breve{\boldsymbol{u}}$ on $\breve{\Omega}$ depends on $(r, z)$. For any vector field $\breve{\boldsymbol{u}}$, we denote by $\breve{u}_{r}, \breve{u}_{\theta}, \breve{u}_{z}$ its radial, angular and axial components, which are functions of $r$ and $z$, therefore we associate a vector field $\boldsymbol{u}=\left(u_{r}, u_{\theta}, u_{z}\right)$ on $\Omega$ such that $u_{r}=\breve{u}_{r}, u_{\theta}=\breve{u}_{\theta}$, and $u_{z}=\breve{u}_{z}$.

Relying on the isomorphisms proved in [6, Chap. II], we can write and analyze the variational formulation of the reduced two-dimensional problems. We recall that $f_{r}, f_{\theta}, f_{z}$ denote the cylindrical components of $\boldsymbol{f}$, which are independent of $\theta . p_{b}$ and $g$ are also independent of $\theta$.

We introduce the following operators

$$
\operatorname{grad} p=\left[\begin{array}{c}
\partial_{r} p \\
0 \\
\partial_{z} p
\end{array}\right] \quad \text { and } \quad \operatorname{div} \boldsymbol{u}=\partial_{r} u_{r}+\frac{1}{r} u_{r}+\partial_{z} u_{z}
$$

Then problem (1) is equivalent to

$$
\begin{cases}\partial_{t} u_{r}+\alpha u_{r}+\partial_{r} p=f_{r} & \text { in } \Omega \times] 0, T[, \\ \partial_{t} u_{\theta}+\alpha u_{\theta}=f_{\theta} & \text { in } \Omega \times] 0, T[, \\ \partial_{t} u_{z}+\alpha u_{z}+\partial_{z} p=f_{z} & \text { in } \Omega \times] 0, T[, \\ \partial_{r} u_{r}+r^{-1} u_{r}+\partial_{z} u_{z}=0 & \text { in } \Omega \times] 0, T[, \\ p=p_{b} & \text { on } \left.\Gamma_{p} \times\right] 0, T[, \\ u_{r} n_{r}+u_{z} n_{z}=g & \text { on } \left.\Gamma_{u} \times\right] 0, T[, \\ \left(u_{r}, u_{\theta}, u_{z}\right)=\left(u_{0 r}, u_{0 \theta}, u_{0 z}\right) & \text { in } \Omega \text { at time } t=0 .\end{cases}
$$

This problem reduces to a system of two uncoupled problems that we treat separately: the simplest one is a scalar coercive equation for the angular velocity $u_{\theta}$ and the other is a saddle-point type problem for $\left(u_{r}, u_{z}, p\right)$.

The component $u_{\theta}$ is obtained by applying the variation of constants method, 
yields for a.e. $t, 0 \leq t \leq T$

$$
u_{\theta}(., t)=e^{-\alpha t}\left(\int_{0}^{t} e^{\alpha s} f_{\theta}(., t) d s+u_{0 \theta}\right),
$$

where $u_{\theta}$ belongs to $H^{1}\left(0, T ; L_{1}^{2}(\Omega)\right)$.

So from now on we only consider the reduced problem

$$
\begin{cases}\partial_{t} u_{r}+\alpha u_{r}+\partial_{r} p=f_{r} & \text { in } \Omega \times] 0, T[, \\ \partial_{t} u_{z}+\alpha u_{z}+\partial_{z} p=f_{z} & \text { in } \Omega \times] 0, T[, \\ \partial_{r} u_{r}+r^{-1} u_{r}+\partial_{z} u_{z}=0 & \text { in } \Omega \times] 0, T[, \\ p=p_{b} & \text { on } \left.\Gamma_{p} \times\right] 0, T[, \\ u_{r} n_{r}+u_{z} n_{z}=g & \text { on } \left.\Gamma_{u} \times\right] 0, T[, \\ \left(u_{r}, u_{z}\right)=\left(u_{0 r}, u_{0 z}\right) & \text { in } \Omega \text { at time } t=0 .\end{cases}
$$

In order to write the variational formulation of problem (3) and according to [6, Sec. II.2], we consider the weighted Sobolev spaces

$$
L_{1}^{2}(\Omega)=\left\{v: \Omega \rightarrow \mathbb{R} \text { measurable; } \int_{\Omega}|v(r, z)|^{2} r d r d z<+\infty\right\},
$$

equipped with the norm

$$
\|v\|_{L_{1}^{2}(\Omega)}=\left(\int_{\Omega}|v(r, z)|^{2} r d r d z\right)^{\frac{1}{2}}
$$

and also the space

$$
H_{1}^{1}(\Omega)=\left\{v \in L_{1}^{2}(\Omega) ; \partial_{r} v \in L_{1}^{2}(\Omega) \text { et } \partial_{z} v \in L_{1}^{2}(\Omega)\right\},
$$

which is provided with the seminorm and norm

$$
|v|_{H_{1}^{1}(\Omega)}=\left(\left\|\partial_{z} v\right\|_{L_{1}^{2}(\Omega)}^{2}+\left\|\partial_{r} v\right\|_{L_{1}^{2}(\Omega)}^{2}\right)^{\frac{1}{2}}, \quad\|v\|_{H_{1}^{1}(\Omega)}=\left(\|v\|_{L_{1}^{2}(\Omega)}^{2}+|v|_{H_{1}^{1}(\Omega)}^{2}\right)^{\frac{1}{2}} .
$$

The trace on $\Gamma_{u}$ is defined in a nearly standard way see [5, Sec. 2]. If $H_{1}^{s}\left(\Gamma_{u}\right)$, $s \geq 0$, stands for the scale of Sobolev spaces built from

$$
L_{1}^{2}\left(\Gamma_{u}\right)=\left\{g: \Gamma_{u} \rightarrow \mathbb{R} \text { mesurable; } \int_{\Gamma_{u}} g^{2}(\tau) r(\tau) d \tau<+\infty\right\},
$$

(where $r(\tau)$ denotes the distance of the point with tangential coordinate $\tau$ to the axis $r=0)$, the trace operator: $v \longmapsto v_{\mid \Gamma_{u}}$ is continuous from $H_{1}^{1}(\Omega)$ onto $H_{1}^{\frac{1}{2}}\left(\Gamma_{u}\right)$ see [6, Chap. II].

Let also $H_{1}^{\frac{1}{2}}\left(\Gamma_{p}\right)$ the space of traces of functions in $H_{1}^{1}(\Omega)$ on $\Gamma_{p}$. The variational space

$$
H_{1 \diamond}^{1}(\Omega)=\left\{q \in H_{1}^{1}(\Omega) ; q=0 \text { sur } \Gamma_{p}\right\},
$$


is a Hilbert space for the scalar product associated with the previously defined norm.

We denote by $(., .)_{1}$ the scalar product on $L_{1}^{2}(\Omega)^{2}$.

The variational formulation of the saddlepoint type problem is written:

Find $\left(\boldsymbol{u}=\left(u_{r}, u_{z}\right), p\right)$ in $H^{1}\left(0, T ; L_{1}^{2}(\Omega)^{2}\right) \times L^{2}\left(0, T ; H_{1}^{1}(\Omega)\right)$ such that

$$
\boldsymbol{u}(., 0)=\boldsymbol{u}_{0} \quad \text { in } \Omega,
$$

for a.e. $t, \quad 0 \leq t \leq T$,

$$
\begin{gathered}
p(., t)=p_{b} \quad \text { on } \quad \Gamma_{p}, \\
\forall \boldsymbol{v} \in L_{1}^{2}(\Omega)^{2}, a\left(\partial_{t} \boldsymbol{u}, \boldsymbol{v}\right)+\alpha a(\boldsymbol{u}, \boldsymbol{v})+b(\boldsymbol{v}, p)=\int_{\Omega} \boldsymbol{f}(r, z) \cdot \boldsymbol{v}(r, z) r d r d z, \\
\forall q \in H_{1 \diamond}^{1}(\Omega), b(\boldsymbol{u}, q)=\int_{\Gamma_{u}} g(\tau) q(\tau) r(\tau) d \tau
\end{gathered}
$$

where the bilinear forms $a(.,$.$) and b(.,$.$) are defined by:$

$$
\begin{gathered}
a(\boldsymbol{u}, \boldsymbol{v})=(\boldsymbol{u}, \boldsymbol{v})_{1}=\int_{\Omega}\left(u_{r}(r, z) \cdot v_{r}(r, z)+u_{z}(r, z) \cdot v_{z}(r, z)\right) r d r d z, \\
b(\boldsymbol{v}, q)=(\boldsymbol{v}, \operatorname{grad} q)_{1}=\int_{\Omega}\left(v_{r}(r, z) \cdot \partial_{r} q(r, z)+v_{z}(r, z) \cdot \partial_{z} q(r, z)\right) r d r d z .
\end{gathered}
$$

It is readily checked that the forms $a(.,$.$) and b(.,$.$) are continuous on$ $L_{1}^{2}(\Omega)^{2} \times L_{1}^{2}(\Omega)^{2}$ and $L_{1}^{2}(\Omega)^{2} \times H_{1}^{1}(\Omega)$ respectively.

The kernel of the bilinear form $b(.,$.$) is$

$$
\mathbb{V}(\Omega)=\left\{\boldsymbol{v} \in L_{1}^{2}(\Omega)^{2} ; \forall q \in H_{1 \diamond}^{1}(\Omega), b(\boldsymbol{v}, q)=0\right\},
$$

is characterized by

$$
\mathbb{V}(\Omega)=\left\{\boldsymbol{v} \in L_{1}^{2}(\Omega)^{2} ; \operatorname{div} \boldsymbol{v}=0 \text { and } \boldsymbol{v} . \boldsymbol{n}=0 \text { on } \Gamma_{u}\right\},
$$

and its orthogonal in $L_{1}^{2}(\Omega)^{2}$ is defined by

$$
\mathbb{V}(\Omega)^{\perp}=\left\{\boldsymbol{v} \in L_{1}^{2}(\Omega)^{2} ; \forall \boldsymbol{w} \in \mathbb{V}(\Omega), \int_{\Omega} \boldsymbol{v} \cdot \boldsymbol{w} r d r d z=0\right\} .
$$

Proving the well-posedness of problem (4-5-6) relies on the ellipticity of $a(.,$. and on an inf-sup condition of Babuška and Brezzi type on the form $b(.,$.$) . We$ begin with this condition.

Lemma 1. There exists a constant $\beta>0$ such that the following inf-sup condition holds

$$
\forall q \in H_{1 \diamond}^{1}(\Omega), \quad \sup _{\boldsymbol{v}=\left(v_{r}, v_{z}\right) \in L_{1}^{2}(\Omega)^{2}} \frac{b(\boldsymbol{v}, q)}{\|\boldsymbol{v}\|_{L_{1}^{2}(\Omega)^{2}}} \geq \beta|q|_{H_{1}^{1}(\Omega)} .
$$


Proof. Let $q$ be any function in $H_{1 \diamond}^{1}(\Omega)$. The idea is to choose $\boldsymbol{v}$ equal to $\operatorname{grad} q$, so that

$$
b(\boldsymbol{v}, q)=\left\|\partial_{r} q\right\|_{L_{1}^{2}(\Omega)}^{2}+\left\|\partial_{z} q\right\|_{L_{1}^{2}(\Omega)}^{2}=|q|_{H_{1}^{1}(\Omega)}^{2},
$$

and

$$
\|\boldsymbol{v}\|_{L_{1}^{2}(\Omega)^{2}}=|q|_{H_{1}^{1}(\Omega)} .
$$

This gives the desired inf-sup condition.

To make this condition complete, the weighted Poincaré-Friedrichs condition ensures the equivalence between the norms $|\cdot|_{H_{1}^{1}(\Omega)}$ and $\|\cdot\|_{H_{1}^{1}(\Omega)}$ on $H_{1 \diamond}^{1}(\Omega)$, see [2, Lem. 3.1].

Lemma 2. The following ellipticity property holds

$$
\forall \boldsymbol{v}=\left(v_{r}, v_{z}\right) \in L_{1}^{2}(\Omega)^{2}, \quad a(\boldsymbol{v}, \boldsymbol{v}) \geq\|\boldsymbol{v}\|_{L_{1}^{2}(\Omega)^{2}}^{2} .
$$

Proof. We have

$$
a(\boldsymbol{v}, \boldsymbol{v})=\int_{\Omega}|\boldsymbol{v}(r, z)|^{2} r d r d z=\|\boldsymbol{v}\|_{L_{1}^{2}(\Omega)^{2}}^{2},
$$

which implies the desired inequality.

Thanks to Lemmas 1 and 2, we easily derive the next theorem, see [6].

Theorem 1. For any data

$$
\left(\boldsymbol{f}, p_{b}, g\right) \in L^{2}\left(0, T ; L_{1}^{2}(\Omega)^{2}\right) \times L^{2}\left(0, T ; H_{1}^{\frac{1}{2}}\left(\Gamma_{p}\right)\right) \times L^{2}\left(0, T ; L_{1}^{2}\left(\Gamma_{u}\right)\right)
$$

and $\boldsymbol{u}_{0} \in L_{1}^{2}(\Omega)^{2}$, problem (4-5-6) has a unique solution

$$
\left(\boldsymbol{u}=\left(u_{r}, u_{z}\right), p\right) \in H^{1}\left(0, T ; L_{1}^{2}(\Omega)^{2}\right) \times L^{2}\left(0, T ; H_{1}^{1}(\Omega)\right) .
$$

Moreover the 4-tuple $\left(u_{r}, u_{\theta}, u_{z}, p\right)$ is equal to $\left(R_{-\theta} \breve{\boldsymbol{u}}, \breve{p}\right)$, where $(\breve{\boldsymbol{u}}, \breve{p})$ is the solution of problem (1) with axisymmetic data and $R_{\theta}$ the rotation with angle $\theta$ with respect to the axis $r=0$ in $\mathbb{R}^{3}$.

Remark 1. The third equation in problem (3) can equivalently be written

$$
\partial_{r}\left(r u_{r}\right)+\partial_{z}\left(r u_{z}\right)=0 .
$$

Hence, the function $\left(r u_{r}, r u_{z}\right)$ is divergence-free in the standard sense, so it is the curl of a function $\varphi$. Setting: $\varphi=r \psi$, we derive the existence of a scalar potential $\psi$ such that

$$
u_{r}=\partial_{z} \psi \quad \text { and } \quad u_{z}=-\frac{1}{r} \partial_{r}(r \psi) \quad \text { on } \quad \Omega .
$$


Now we will find a new simpler variationel formulation which is equivalent to problem (4-5-6). For this let $\mathcal{L}$ denote a lifting operator, which is continuous from $H_{1}^{\frac{1}{2}}\left(\Gamma_{p}\right)$ into $H_{1}^{1}(\Omega)$, the existence of such operateur is established in [12], for instance. Since $p_{b} \in L^{2}\left(0, T ; H_{1}^{\frac{1}{2}}\left(\Gamma_{p}\right)\right)$ we denote by $\bar{p}_{b}$ the function defined for a.e. $t, 0 \leq t \leq T$,

$$
\bar{p}_{b}(t)=\mathcal{L}\left(p_{b}(t)\right) .
$$

The function $\bar{p}_{b}$ belongs to $L^{2}\left(0, T ; H_{1}^{1}(\Omega)\right)$ and satisfies

$$
\left\|\bar{p}_{b}\right\|_{L^{2}\left(0, T ; H_{1}^{1}(\Omega)\right)} \leq c_{0}\left\|p_{b}\right\|_{L^{2}\left(0, T ; H_{1}^{\frac{1}{2}}\left(\Gamma_{p}\right)\right)} .
$$

The last equation in problem (4-5-6) can be viewed as a non-homogeneous constraint; let us show that we can find a function of $L_{1}^{2}(\Omega)^{2}$ that satisfies this constraint. For this, define the linear operator $B: L_{1}^{2}(\Omega)^{2} \rightarrow H_{1 \diamond}^{1}(\Omega)^{\prime}$ by

$$
\langle B \boldsymbol{v}, q\rangle_{H_{1 \diamond}^{1}(\Omega)^{\prime} \times H_{1 \diamond}^{1}(\Omega)}=b(\boldsymbol{v}, q) .
$$

It follows from inf-sup condition (7) and [10, Chap. I, Lem. 4.1], that this operator is an isomorphism from $\mathbb{V}(\Omega)^{\perp}$ onto $H_{1 \diamond}^{1}(\Omega)^{\prime}$ and

$$
\forall \boldsymbol{v} \in \mathbb{V}(\Omega)^{\perp}, \quad\|B \boldsymbol{v}\|_{H_{1 \diamond}^{1}(\Omega)^{\prime}} \geq \beta\|\boldsymbol{v}\|_{L_{1}^{2}(\Omega)^{2}} .
$$

Hence, for $g \in H_{1 \diamond}^{1}(\Omega)^{\prime}$ there exists a unique $\boldsymbol{u}_{b} \in \mathbb{V}(\Omega)^{\perp}$ such that

$$
\forall q \in H_{1 \diamond}^{1}(\Omega), \quad b\left(\boldsymbol{u}_{b}, q\right)=\langle g, q\rangle_{\Gamma_{u}},
$$

and

$$
\beta\left\|\boldsymbol{u}_{b}(., t)\right\|_{L_{1}^{2}(\Omega)^{2}} \leq\|g(., t)\|_{L_{1}^{2}\left(\Gamma_{u}\right)} .
$$

When setting $\boldsymbol{u}_{\diamond}=\boldsymbol{u}-\boldsymbol{u}_{b}$ and $p_{*}=p-\bar{p}_{b}$, we observe that

$$
\boldsymbol{u}_{\diamond} \in H^{1}(0, T ; \mathbb{V}(\Omega)) \quad \text { and } \quad p_{*} \in L^{2}\left(0, T ; H_{1 \diamond}^{1}(\Omega)\right) .
$$

Moreover, if $(\boldsymbol{u}, p)$ is a solution of (4-5-6) then $\boldsymbol{u}_{\diamond} \in H^{1}(0, T ; \mathbb{V}(\Omega))$ is the unique solution of the simpler variational problem:

Find $\boldsymbol{u}_{\diamond}=\left(\boldsymbol{u}_{r \diamond}, \boldsymbol{u}_{z \diamond}\right)$ in $H^{1}(0, T ; \mathbb{V}(\Omega))$ such that, for a.e. $t, 0 \leq t \leq T$,

$$
\left\{\begin{array}{l}
\boldsymbol{u}_{\diamond}(., 0)=\boldsymbol{u}_{0}-\boldsymbol{u}_{b 0}=\boldsymbol{u}_{\diamond 0} \text { in } \Omega, \\
\forall \boldsymbol{v} \in \mathbb{V}(\Omega) ;\left(\partial_{t} \boldsymbol{u}_{\diamond}, \boldsymbol{v}\right)_{1}+\alpha\left(\boldsymbol{u}_{\diamond}, \boldsymbol{v}\right)_{1}=(\boldsymbol{f}, \boldsymbol{v})_{1}-\left(\boldsymbol{v}, \operatorname{grad} \bar{p}_{b}\right)_{1},
\end{array}\right.
$$

where $\boldsymbol{u}_{b 0}=\boldsymbol{u}_{b}(., 0)$ in $\Omega$.

Conversely, let us prove that, if $\boldsymbol{u}_{\diamond}$ is a solution of (13), then there exists a unique pair $\left(\boldsymbol{u}=\boldsymbol{u}_{\diamond}+\boldsymbol{u}_{b}, p=p_{*}+\bar{p}_{b}\right)$ in $H^{1}\left(0, T ; L_{1}^{2}(\Omega)^{2}\right) \times L^{2}\left(0, T ; H_{1}^{1}(\Omega)\right)$ solution of problem (4-5-6). For this, we integrate the second equation in (13) between 0 and $t$, we define the functional for all $\boldsymbol{v} \in L_{1}^{2}(\Omega)^{2}$ :

$L_{t}(\boldsymbol{v})=\int_{0}^{t}\left((\boldsymbol{f}(., s), \boldsymbol{v})_{1}-\alpha\left(\boldsymbol{u}_{\diamond}(., s), \boldsymbol{v}\right)_{1}-b\left(\boldsymbol{v}, \bar{p}_{b}(s)\right)\right) d s-\left(\boldsymbol{u}_{\diamond}(., t), \boldsymbol{v}\right)_{1}+\left(\boldsymbol{u}_{\diamond 0}, \boldsymbol{v}\right)_{1}$. 
For all $t \in[0, T], L_{t}$ is a continuous linear functional on $L_{1}^{2}(\Omega)^{2}$ and, according to (13), $L_{t}(\boldsymbol{v})=0 \forall \boldsymbol{v} \in \mathbb{V}(\Omega)$. Hence, see [9, Chap. V, Thm. 1.3], for each $t \in[0, T]$, there exists a unique function $P(t)$ in $H_{1 \diamond}^{1}(\Omega)$ such that:

$$
\begin{gathered}
\forall \boldsymbol{v} \in L_{1}^{2}(\Omega)^{2}, L_{t}(\boldsymbol{v})=b(\boldsymbol{v}, P(t)), \\
|P(t)|_{H_{1}^{1}(\Omega)} \leq \sup _{\boldsymbol{v} \in L_{1}^{2}(\Omega)^{2}} \frac{L_{t}(\boldsymbol{v})}{\|\boldsymbol{v}\|_{L_{1}^{2}(\Omega)^{2}}} .
\end{gathered}
$$

Now, differentiating (14) with respect to $t$, and setting $p_{*}(t)=\frac{d}{d t} P(t)$, we obtain (6) with $p=p_{*}+\bar{p}_{b}$. This gives immediately (5).

Then we can conclude the following corollary.

Corollary 1. The variational problems (4-5-6) and (13) are equivalent.

Lemma 3. For any data

$$
\left(\boldsymbol{f}, p_{b}, g\right) \in L^{2}\left(0, T ; L_{1}^{2}(\Omega)^{2}\right) \times L^{2}\left(0, T ; H_{1}^{\frac{1}{2}}\left(\Gamma_{p}\right)\right) \times L^{2}\left(0, T ; L_{1}^{2}\left(\Gamma_{u}\right)\right)
$$

and $\boldsymbol{u}_{0} \in L_{1}^{2}(\Omega)^{2}$, we have the following a priori estimates for the velocity $\boldsymbol{u}$ solution of problem (4-5-6), for a.e. $t \in[0, T]$,

$$
\begin{aligned}
\|\boldsymbol{u}\|_{L^{\infty}\left(0, t ; L_{1}^{2}(\Omega)^{2}\right)} & \leq c\left(\left\|\boldsymbol{u}_{0}\right\|_{L_{1}^{2}(\Omega)^{2}}+\|g(., 0)\|_{L_{1}^{2}\left(\Gamma_{u}\right)}+\|\boldsymbol{f}\|_{L^{2}\left(0, t ; L_{1}^{2}(\Omega)^{2}\right)}\right. \\
& \left.+\left\|p_{b}\right\|_{L^{2}\left(0, t ; H_{1}^{\frac{1}{2}}\left(\Gamma_{p}\right)\right)}+\|g\|_{L^{\infty}\left(0, t ; L_{1}^{2}\left(\Gamma_{u}\right)\right)}\right),
\end{aligned}
$$

with a constant $c$ that only depends on $\Omega$ and $T$.

Proof. Taking $\boldsymbol{v}=\boldsymbol{u}_{\diamond}$ in (13) gives

$\left(\partial_{t} \boldsymbol{u}_{\diamond}, \boldsymbol{u}_{\diamond}\right)_{1}+\alpha\left(\boldsymbol{u}_{\diamond}, \boldsymbol{u}_{\diamond}\right)_{1}=\left(\boldsymbol{f}, \boldsymbol{u}_{\diamond}\right)_{1}-\left(\boldsymbol{u}_{\diamond}, \operatorname{grad} \bar{p}_{b}\right)_{1}$.

By using Cauchy-Schwarz inequality yields

$\frac{1}{2} \frac{d}{d t}\left\|\boldsymbol{u}_{\diamond}\right\|_{L_{1}^{2}(\Omega)^{2}}^{2}+\alpha\left\|\boldsymbol{u}_{\diamond}\right\|_{L_{1}^{2}(\Omega)^{2}}^{2} \leq\left\|\boldsymbol{f}_{L_{1}^{2}(\Omega)^{2}} \cdot\right\| \boldsymbol{u}_{\diamond}\left\|_{L_{1}^{2}(\Omega)^{2}}+\left|\bar{p}_{b}\right|_{H_{1}^{1}(\Omega)} \cdot\right\| \boldsymbol{u}_{\diamond} \|_{L_{1}^{2}(\Omega)^{2}}$.

Using Young's inequality: $a b \leq \frac{a^{2}}{2 \alpha}+\frac{\alpha}{2} b^{2}$, we obtain:

$\frac{d}{d t}\left\|\boldsymbol{u}_{\diamond}\right\|_{L_{1}^{2}(\Omega)^{2}}^{2} \leq \frac{1}{\alpha}\left(\|f\|_{L_{1}^{2}(\Omega)^{2}}^{2}+\left|\bar{p}_{b}\right|_{H_{1}^{1}(\Omega)}^{2}\right)$.

Integrating this inequality between 0 and $t$, using the fact that $\boldsymbol{u}_{\diamond}=\boldsymbol{u}-\boldsymbol{u}_{b}$ in $\Omega$ and $\boldsymbol{u}_{\diamond}(., 0)=\boldsymbol{u}_{0}-\boldsymbol{u}_{b 0}$ yields

$\left\|\boldsymbol{u}(., t)-\boldsymbol{u}_{b}(., t)\right\|_{L_{1}^{2}(\Omega)^{2}}^{2} \leq\left\|\boldsymbol{u}_{0}-\boldsymbol{u}_{b 0}\right\|_{L_{1}^{2}(\Omega)^{2}}^{2}$

$$
+\frac{1}{\alpha}\left(\|\boldsymbol{f}(., t)\|_{L^{2}\left(0, t ; L_{1}^{2}(\Omega)^{2}\right)}^{2}+\left|\bar{p}_{b}(., t)\right|_{L^{2}\left(0, t ; H_{1}^{1}(\Omega)\right)}^{2}\right) .
$$

By triangle inequality, estimates (10) and (12) we obtain

$$
\begin{aligned}
\|\boldsymbol{u}(., t)\|_{L_{1}^{2}(\Omega)^{2}}^{2} \leq & c\left(\left\|\boldsymbol{u}_{0}\right\|_{L_{1}^{2}(\Omega)^{2}}+\|g(., 0)\|_{L_{1}^{2}\left(\Gamma_{u}\right)}+\|\boldsymbol{f}\|_{L^{2}\left(0, t ; L_{1}^{2}(\Omega)^{2}\right)}\right. \\
& \left.+\left\|p_{b}\right\|_{L^{2}\left(0, t ; H_{1}^{\frac{1}{2}}\left(\Gamma_{p}\right)\right)}+\|g(., t)\|_{L_{1}^{2}\left(\Gamma_{u}\right)}\right) .
\end{aligned}
$$

This gives the desired estimate (15).

We refer to [7, Thm. 2.4], for the detailed proof of the next Theorem. 
Theorem 2. For any data

$$
\left(\boldsymbol{f}, p_{b}, g\right) \in L^{2}\left(0, T ; L_{1}^{2}(\Omega)^{2}\right) \times L^{2}\left(0, T ; H_{1}^{\frac{1}{2}}\left(\Gamma_{p}\right)\right) \times H^{1}\left(0, T ; L_{1}^{2}\left(\Gamma_{u}\right)\right)
$$

and $\boldsymbol{u}_{0} \in L_{1}^{2}(\Omega)^{2}$, the unique solution

$$
\left(\boldsymbol{u}=\left(u_{r}, u_{z}\right), p\right) \in H^{1}\left(0, T ; L_{1}^{2}(\Omega)^{2}\right) \times L^{2}\left(0, T ; H_{1}^{1}(\Omega)\right)
$$

of problem (4-5-6), satisfies the a priori estimate for a.e. $t \in[0, T]$,

$$
\begin{aligned}
& \|\boldsymbol{u}\|_{H^{1}\left(0, T ; L_{1}^{2}(\Omega)^{2}\right)}+\|p\|_{L^{2}\left(0, T ; H_{1}^{1}(\Omega)\right)} \\
\leq & c\left(\left\|\boldsymbol{u}_{0}\right\|_{L_{1}^{2}(\Omega)^{2}}+\|\boldsymbol{f}\|_{L^{2}\left(0, T ; L_{1}^{2}(\Omega)^{2}\right)}+\left\|p_{b}\right\|_{L^{2}\left(0, t ; H_{1}^{\frac{1}{2}}\left(\Gamma_{p}\right)\right)}+\|g\|_{H^{1}\left(0, t ; L_{1}^{2}\left(\Gamma_{u}\right)\right)}\right) .
\end{aligned}
$$

\section{The discrete problem and its a priori analysis}

We split the discretization into two steps: First a semi-discretization in time, and next the full discretization. At each step, we prove a priori error estimates.

\subsection{The time semi-discrete problem}

We introduce a partition of the interval $[0, T]$ into subintervals $\left[t_{n-1}, t_{n}\right]$, $1 \leq n \leq N$, such that $0=t_{0}<t_{1}<\ldots<t_{N}=T$. We denote by $\tau_{n}$ the time step $t_{n}-t_{n-1}$, by $\tau$ the $N$ - tuple $\left(\tau_{1}, \tau_{2}, \ldots, \tau_{N}\right)$ and by $|\tau|$ the maximum of the $\tau_{n}, 1 \leq n \leq N$. The time discretization of problem (4-5-6) relies on the use of a backward Euler's scheme.

Thus for any data $\left(\boldsymbol{f}, p_{b}\right) \in C^{0}\left(0, T ; L_{1}^{2}(\Omega)^{2}\right) \times C^{0}\left(0, T ; H_{1}^{\frac{1}{2}}\left(\Gamma_{p}\right)\right)$,

$g \in C^{0}\left(0, T ; L_{1}^{2}\left(\Gamma_{u}\right)\right)$ and $\boldsymbol{u}_{0} \in L_{1}^{2}(\Omega)^{2}$, satisfaying $\operatorname{div} \boldsymbol{u}_{0}=0$ in $\Omega$, we consider the following scheme:

Find $\left(\boldsymbol{u}^{n}\right)_{0 \leq n \leq N} \in\left(L_{1}^{2}(\Omega)^{2}\right)^{N+1}$ and $\left(p^{n}\right)_{1 \leq n \leq N} \in\left(H_{1}^{1}(\Omega)\right)^{N}$ such that

$$
\boldsymbol{u}^{0}=\boldsymbol{u}_{0} \quad \text { in } \Omega,
$$

$\forall n, 1 \leq n \leq N$

$$
\begin{aligned}
& p^{n}=p_{b}^{n} \quad \text { on } \quad \Gamma_{p}, \\
& \forall \boldsymbol{v} \in L_{1}^{2}(\Omega)^{2},\left(\boldsymbol{u}^{n}, \boldsymbol{v}\right)_{1}+\alpha \tau_{n}\left(\boldsymbol{u}^{n}, \boldsymbol{v}\right)_{1}=\left(\boldsymbol{u}^{n-1}, \boldsymbol{v}\right)_{1}-\tau_{n}\left(\boldsymbol{v}, \operatorname{grad} p^{n}\right)_{1} \\
& +\tau_{n}\left(\boldsymbol{f}^{n}, \boldsymbol{v}\right)_{1} \text {, } \\
& \forall q \in H_{1 \diamond}^{1}(\Omega),\left(\boldsymbol{u}^{n}, \operatorname{grad} q\right)_{1}=\left\langle g^{n}, q\right\rangle_{\Gamma_{u}},
\end{aligned}
$$

Now we will find a new simpler variational formulation which is equivalent to problem (18-19-20). For this we use the lifting operator $\mathcal{L}$ introduced in (9), verifying

$$
\left\|\bar{p}_{b}^{n}\right\|_{H_{1}^{1}(\Omega)} \leq c_{0}\left\|p_{b}^{n}\right\|_{H_{1}^{\frac{1}{2}}\left(\Gamma_{P}\right)},
$$


and the linear operator $B$ introduced in (11) which is an isomorphism from $\mathbb{V}(\Omega)^{\perp}$ into $H_{1 \diamond}^{1}(\Omega)^{\prime}$. Hence, there exists a unique $\boldsymbol{u}_{b}^{n} \in \mathbb{V}(\Omega)^{\perp}$ such that

$$
\forall q \in H_{1 \diamond}^{1}(\Omega), \quad b\left(u_{b}^{n}, q\right)=\left\langle g^{n}, q\right\rangle_{\Gamma_{u}},
$$

and

$$
\beta\left\|\boldsymbol{u}_{b}^{n}(., t)\right\|_{L_{1}^{2}(\Omega)^{2}} \leq\left\|g^{n}(., t)\right\|_{L_{1}^{2}\left(\Gamma_{u}\right)} .
$$

When setting $\boldsymbol{u}_{\diamond}^{n}=\boldsymbol{u}^{n}-\boldsymbol{u}_{b}^{n}$, then $\boldsymbol{u}_{\diamond}^{n}$ belongs to $\mathbb{V}(\Omega)$, and the sequence $\left(\boldsymbol{u}_{\diamond}^{n}\right)_{0 \leq n \leq N} \in(\mathbb{V}(\Omega))^{N+1}$ is a solution of the simpler variational problem:

Find $\left(\boldsymbol{u}_{\diamond}^{n}\right)_{0 \leq n \leq N}$ in $(\mathbb{V}(\Omega))^{N+1}$ such that for all $n, 1 \leq n \leq N$,

$$
\left\{\begin{array}{l}
\boldsymbol{u}_{\diamond}^{0}=\boldsymbol{u}^{0}-\boldsymbol{u}_{b}^{0} \quad \text { in } \quad \Omega, \\
\forall \boldsymbol{v} \in \mathbb{V}(\Omega), \\
\left(\boldsymbol{u}_{\diamond}^{n}, \boldsymbol{v}\right)_{1}+\alpha \tau_{n}\left(\boldsymbol{u}_{\diamond}^{n}, \boldsymbol{v}\right)_{1}=\left(\boldsymbol{u}_{\diamond}^{n-1}, \boldsymbol{v}\right)_{1}+\tau_{n}\left(\boldsymbol{f}^{n}, \boldsymbol{v}\right)_{1}-\tau_{n} b\left(\boldsymbol{v}, \bar{p}_{b}^{n}\right) .
\end{array}\right.
$$

Conversely, if $\left(\boldsymbol{u}_{\diamond}^{n}\right)_{0 \leq n \leq N}$ is a solution of (23), we define the linear mapping

$$
L_{n}(\boldsymbol{v})=\int_{\Omega}\left(\boldsymbol{f}^{n} \cdot \boldsymbol{v}\right)(r, z) r d r d z-b\left(\boldsymbol{v}, \bar{p}_{b}^{n}\right)-\frac{1}{\tau_{n}}\left(\boldsymbol{u}_{\diamond}^{n}-\boldsymbol{u}_{\diamond}^{n-1}, \boldsymbol{v}\right)_{1}-\alpha\left(\boldsymbol{u}_{\diamond}^{n}, \boldsymbol{v}\right)_{1} .
$$

The mapping: $\boldsymbol{v} \mapsto L_{n}(\boldsymbol{v})$ is a continuous linear functional on $L_{1}^{2}(\Omega)^{2}$, and $\forall \boldsymbol{v} \in \mathbb{V}(\Omega) L_{n}(\boldsymbol{v})=0$, thus the inf-sup condition (7) implies that there exists a unique element $p_{*}^{n}(t)$ in $H_{1 \diamond}^{1}(\Omega)$ such that:

$$
\begin{aligned}
& \forall \boldsymbol{v} \in L_{1}^{2}(\Omega)^{2}, b\left(\boldsymbol{v}, p_{*}^{n}\right)=L_{n}(\boldsymbol{v}), \\
& \left|p_{*}^{n}\right|_{H_{1}^{1}(\Omega)} \leq \sup _{\boldsymbol{v} \in L_{1}^{2}(\Omega)^{2}} \frac{L_{n}(\boldsymbol{v})}{\|\boldsymbol{v}\|_{L_{1}^{2}(\Omega)^{2}}} .
\end{aligned}
$$

Therefore, $\left(\boldsymbol{u}^{n}=\boldsymbol{u}_{\diamond}^{n}+\boldsymbol{u}_{b}^{n}, p^{n}=p_{*}^{n}+\bar{p}_{b}^{n}\right)$ is a solution of problem (18-19-20). Then we can conclude the following lemma:

Lemma 4. The variational problems (18-19-20) and (23) are equivalent.

Theorem 3. For any data $\left(\boldsymbol{f}, p_{b}\right) \in C^{0}\left(0, T ; L_{1}^{2}(\Omega)^{2}\right) \times C^{0}\left(0, T ; H_{1}^{\frac{1}{2}}\left(\Gamma_{p}\right)\right)$, $g \in L^{2}\left(0, T ; L_{1}^{2}\left(\Gamma_{u}\right)\right)$ and $\boldsymbol{u}_{0} \in L_{1}^{2}(\Omega)^{2}$, satisfaying $\operatorname{div} \boldsymbol{u}_{0}=0$ in $\Omega$, problem (18-19-20) has a unique solution $\left(\boldsymbol{u}^{n}, p^{n}\right)$ such that:

$$
\forall n, 0 \leq n \leq N, \boldsymbol{u}^{n} \in L_{1}^{2}(\Omega)^{2} \text { and } \forall n, 1 \leq n \leq N, p^{n} \in H_{1}^{1}(\Omega) .
$$

Moreover the sequence of velocities $\left(\boldsymbol{u}^{n}\right)_{0 \leq n \leq N}$ satisfies:

$$
\begin{gathered}
\left\|\boldsymbol{u}^{n}\right\|_{L_{1}^{2}(\Omega)^{2}} \leq\left\|\boldsymbol{u}_{0}\right\|_{L_{1}^{2}(\Omega)^{2}}+\sqrt{\frac{2}{\alpha}}\left(\sum_{m=1}^{n} \tau_{m}\left(\left\|\boldsymbol{f}^{m}\right\|_{L_{1}^{2}(\Omega)^{2}}^{2}+c_{0}^{2}\left\|p_{b}^{m}\right\|_{H_{1}^{\frac{1}{2}}\left(\Gamma_{p}\right)}^{2}\right)\right)^{\frac{1}{2}} \\
+\left\|g^{n}\right\|_{L_{1}^{2}\left(\Gamma_{u}\right)}+\|g(., 0)\|_{L_{1}^{2}\left(\Gamma_{u}\right)},
\end{gathered}
$$




$$
\begin{aligned}
\left(\sum_{m=1}^{n} \tau_{m}\left\|\frac{\boldsymbol{u}^{m}-\boldsymbol{u}^{m-1}}{\tau_{m}}\right\|_{L_{1}^{2}(\Omega)^{2}}^{2}\right. & )^{\frac{1}{2}} \leq \sqrt{2 \alpha}\left(\left\|\boldsymbol{u}_{0}\right\|_{L_{1}^{2}(\Omega)^{2}}+\|g(., 0)\|_{L_{1}^{2}\left(\Gamma_{u}\right)}\right) \\
+ & \left(\sum_{m=1}^{n} \tau_{m}\left(\left\|\boldsymbol{f}^{m}\right\|_{L_{1}^{2}(\Omega)^{2}}^{2}+c_{0}^{2}\left\|p_{b}^{m}\right\|_{H_{1}^{\frac{1}{2}}\left(\Gamma_{p}\right)}^{2}\right)\right)^{\frac{1}{2}} \\
+ & \frac{\sqrt{2}}{\beta}\left(\sum_{m=1}^{n} \frac{1}{\tau_{m}}\left\|g^{m}-g^{m-1}\right\|_{L_{1}^{2}\left(\Gamma_{u}\right)}^{2}\right)^{\frac{1}{2}} .
\end{aligned}
$$

And the sequence of pressures $\left(p^{n}\right)_{1 \leq n \leq N}$ satisfies:

$$
\begin{aligned}
\left(\sum_{m=1}^{n} \tau_{m}\left|p^{m}\right|_{H_{1}^{1}(\Omega)}^{2}\right)^{\frac{1}{2}} & \leq c\left(\left\|\boldsymbol{u}_{0}\right\|_{L_{1}^{2}(\Omega)^{2}}^{2}+\sum_{m=1}^{n} \tau_{m}\left(\left\|\boldsymbol{f}^{m}\right\|_{L_{1}^{2}(\Omega)^{2}}^{2}+\left\|p_{b}^{m}\right\|_{H_{1}^{\frac{1}{2}}\left(\Gamma_{p}\right)}^{2}\right)\right. \\
& \left.\left.+\|g(., 0)\|_{L_{1}^{2}\left(\Gamma_{u}\right)}\right)+\sum_{m=1}^{n} \frac{1}{\tau_{m}}\left\|g^{m}-g^{m-1}\right\|_{L_{1}^{2}\left(\Gamma_{u}\right)}^{2}\right)^{\frac{1}{2}} \cdot
\end{aligned}
$$

Proof. Clearly, problem (23) has a unique solution: $\boldsymbol{u}_{\diamond}^{n}=\boldsymbol{u}^{n}-\boldsymbol{u}_{b}^{n}$ in $\mathbb{V}(\Omega)$, which yields the existence and uniqueness of the solution $\left(\boldsymbol{u}^{n}=\boldsymbol{u}_{\diamond}^{n}+\boldsymbol{u}_{b}^{n}, p^{n}=p_{*}^{n}+\bar{p}_{b}^{n}\right)$ of problem (18-19-20).

1) To prove estimate (24), we choose $\boldsymbol{v}=\boldsymbol{u}_{\diamond}^{n}$ in (23), this gives $\left\|\boldsymbol{u}_{\diamond}^{n}\right\|_{L_{1}^{2}(\Omega)^{2}}^{2}-\left(\boldsymbol{u}_{\diamond}^{n-1}, \boldsymbol{u}_{\diamond}^{n}\right)_{1}+\alpha \tau_{n}\left\|\boldsymbol{u}_{\diamond}^{n}\right\|_{L_{1}^{2}(\Omega)^{2}}^{2}=\tau_{n}\left(\boldsymbol{f}^{n}, \boldsymbol{u}_{\diamond}^{n}\right)_{1}-\tau_{n}\left(\boldsymbol{u}_{\diamond}^{n}, \operatorname{grad} \bar{p}_{b}^{n}\right)_{1}$. Using the fact that $\left(a^{2}-a b\right)=\frac{1}{2}\left(a^{2}-b^{2}+(a-b)^{2}\right)$ and the Cauchy-Schwarz inequality we obtain

$$
\begin{aligned}
& \frac{1}{2}\left(\left\|\boldsymbol{u}_{\diamond}^{n}\right\|_{L_{1}^{2}(\Omega)^{2}}^{2}-\left\|\boldsymbol{u}_{\diamond}^{n-1}\right\|_{L_{1}^{2}(\Omega)^{2}}^{2}+\left\|\boldsymbol{u}_{\diamond}^{n}-\boldsymbol{u}_{\diamond}^{n-1}\right\|_{L_{1}^{2}(\Omega)^{2}}^{2}\right)+\alpha \tau_{n}\left\|\boldsymbol{u}_{\diamond}^{n}\right\|_{L_{1}^{2}(\Omega)^{2}}^{2} \\
& \leq \tau_{n}\left(\left\|\boldsymbol{f}^{n}\right\|_{L_{1}^{2}(\Omega)^{2}}+\left|\bar{p}_{b}^{n}\right|_{H_{1}^{1}(\Omega)}\right)\left\|\boldsymbol{u}_{\diamond}^{n}\right\|_{L_{1}^{2}(\Omega)^{2}} .
\end{aligned}
$$

Therefore, Young's inequality gives

$$
\begin{aligned}
& \left\|\boldsymbol{u}_{\diamond}^{n}\right\|_{L_{1}^{2}(\Omega)^{2}}^{2}-\left\|\boldsymbol{u}_{\diamond}^{n-1}\right\|_{L_{1}^{2}(\Omega)^{2}}^{2}+\left\|\boldsymbol{u}_{\diamond}^{n}-\boldsymbol{u}_{\diamond}^{n-1}\right\|_{L_{1}^{2}(\Omega)^{2}}^{2}+\alpha \tau_{n}\left\|\boldsymbol{u}_{\diamond}^{n}\right\|_{L_{1}^{2}(\Omega)^{2}}^{2} \\
& \leq \frac{2 \tau_{n}}{\alpha}\left(\left\|\boldsymbol{f}^{n}\right\|_{L_{1}^{2}(\Omega)^{2}}^{2}+\left|\bar{p}_{b}^{n}\right|_{H_{1}^{1}(\Omega)}^{2}\right) .
\end{aligned}
$$

Summing this inequality over $n, 1 \leq n \leq N$ we obtain

$$
\begin{array}{r}
\left\|\boldsymbol{u}_{\diamond}^{n}\right\|_{L_{1}^{2}(\Omega)^{2}}^{2}+\sum_{m=1}^{n}\left\|\boldsymbol{u}_{\diamond}^{m}-\boldsymbol{u}_{\diamond}^{m-1}\right\|_{L_{1}^{2}(\Omega)^{2}}^{2}+\alpha \sum_{m=1}^{n} \tau_{m}\left\|\boldsymbol{u}_{\diamond}^{m}\right\|_{L_{1}^{2}(\Omega)^{2}}^{2} \\
\leq\left\|\boldsymbol{u}_{\diamond}^{0}\right\|_{L_{1}^{2}(\Omega)^{2}}^{2}+\frac{2}{\alpha} \sum_{m=1}^{n} \tau_{m}\left(\left\|\boldsymbol{f}^{m}\right\|_{L_{1}^{2}(\Omega)^{2}}^{2}+\left|\bar{p}_{b}^{m}\right|_{H_{1}^{1}(\Omega)}^{2}\right),
\end{array}
$$

then,

$$
\left\|\boldsymbol{u}_{\diamond}^{n}\right\|_{L_{1}^{2}(\Omega)^{2}} \leq\left\|\boldsymbol{u}_{\diamond}^{0}\right\|_{L_{1}^{2}(\Omega)^{2}}+\sqrt{\frac{2}{\alpha}}\left(\sum_{m=1}^{n} \tau_{m}\left(\left\|\boldsymbol{f}^{m}\right\|_{L_{1}^{2}(\Omega)^{2}}^{2}+\left\|\bar{p}_{b}^{m}\right\|_{H_{1}^{1}(\Omega)}^{2}\right)\right)^{\frac{1}{2}} .
$$


Therefore by using the triangle inequality, the fact that $\boldsymbol{u}_{\diamond}^{n}=\boldsymbol{u}^{n}-\boldsymbol{u}_{b}^{n}$, the initial condition of problem (23), the estimates (21) and (22), we obtain the desired estimate (24).

2) In order to prove estimate (25), we take $\boldsymbol{v}=\left(\boldsymbol{u}_{\diamond}^{n}-\boldsymbol{u}_{\diamond}^{n-1}\right) \in \mathbb{V}(\Omega)$ in problem (23), yields

$$
\begin{aligned}
\left\|\boldsymbol{u}_{\diamond}^{n}-\boldsymbol{u}_{\diamond}^{n-1}\right\|_{L_{1}^{2}(\Omega)^{2}}^{2}+\alpha \tau_{n}\left(\left\|\boldsymbol{u}_{\diamond}^{n}\right\|_{L_{1}^{2}(\Omega)^{2}}^{2}-\left(\boldsymbol{u}_{\diamond}^{n}, \boldsymbol{u}_{\diamond}^{n-1}\right)_{1}\right) & =\tau_{n}\left(\boldsymbol{f}^{n}, \boldsymbol{u}_{\diamond}^{n}-\boldsymbol{u}_{\diamond}^{n-1}\right)_{1} \\
& -\tau_{n}\left(\boldsymbol{u}_{\diamond}^{n}-\boldsymbol{u}_{\diamond}^{n-1}, \operatorname{grad} \bar{p}_{b}^{n}\right)_{1} .
\end{aligned}
$$

By using the fact that $\left(a^{2}-a b\right)=\frac{1}{2}\left(a^{2}-b^{2}+(a-b)^{2}\right)$ and Cauchy-Schwarz inequality we obtain

$\frac{1}{2}\left\|\boldsymbol{u}_{\diamond}^{n}-\boldsymbol{u}_{\diamond}^{n-1}\right\|_{L_{1}^{2}(\Omega)^{2}}^{2}+\frac{\alpha \tau_{n}}{2}\left(\left\|\boldsymbol{u}_{\diamond}^{n}\right\|_{L_{1}^{2}(\Omega)^{2}}^{2}-\left\|\boldsymbol{u}_{\diamond}^{n-1}\right\|_{L_{1}^{2}(\Omega)^{2}}^{2}+\left\|\boldsymbol{u}_{\diamond}^{n}-\boldsymbol{u}_{\diamond}^{n-1}\right\|_{L_{1}^{2}(\Omega)^{2}}^{2}\right)$ $\leq \tau_{n}^{2}\left(\left\|\boldsymbol{f}^{n}\right\|_{L_{1}^{2}(\Omega)^{2}}^{2}+\left|\bar{p}_{b}^{n}\right|_{H_{1}^{1}(\Omega)}^{2}\right)$.

Multiplying by $\frac{2}{\tau_{n}}$ and summing over $n$, with $1 \leq n \leq N$ yields

$$
\begin{gathered}
\sum_{m=1}^{n} \tau_{m}\left\|\frac{\boldsymbol{u}_{\diamond}^{m}-\boldsymbol{u}_{\diamond}^{m-1}}{\tau_{m}}\right\|_{L_{1}^{2}(\Omega)^{2}}^{2}+\alpha \sum_{m=1}^{n}\left\|\boldsymbol{u}_{\diamond}^{m}-\boldsymbol{u}_{\diamond}^{m-1}\right\|_{L_{1}^{2}(\Omega)^{2}}^{2}-\alpha\left\|\boldsymbol{u}_{\diamond}^{0}\right\|_{L_{1}^{2}(\Omega)^{2}}^{2} \\
\leq 2 \sum_{m=1}^{n} \tau_{m}\left(\left\|\boldsymbol{f}^{m}\right\|_{L_{1}^{2}(\Omega)^{2}}^{2}+\left|\bar{p}_{b}^{m}\right|_{H_{1}^{1}(\Omega)}^{2}\right)
\end{gathered}
$$

whence,

$$
\begin{aligned}
\sum_{m=1}^{n} \tau_{m}\left\|\frac{\boldsymbol{u}_{\diamond}^{m}-\boldsymbol{u}_{\diamond}^{m-1}}{\tau_{m}}\right\|_{L_{1}^{2}(\Omega)^{2}}^{2} & \leq \alpha\left\|\boldsymbol{u}_{\diamond}^{0}\right\|_{L_{1}^{2}(\Omega)^{2}}^{2} \\
& +2 \sum_{m=1}^{n} \tau_{m}\left(\left\|\boldsymbol{f}^{m}\right\|_{L_{1}^{2}(\Omega)^{2}}^{2}+\left\|\bar{p}_{b}^{m}\right\|_{H_{1}^{1}(\Omega)}^{2}\right) .
\end{aligned}
$$

On the other hand by using the triangle inequatity, the fact that $\boldsymbol{u}_{\diamond}^{n}=\boldsymbol{u}^{n}-\boldsymbol{u}_{b}^{n}$ for any $n, 0 \leq n \leq N$, and estimate (22), we obtain $\left\|\boldsymbol{u}^{n}-\boldsymbol{u}^{n-1}\right\|_{L_{1}^{2}(\Omega)^{2}} \leq\left\|\boldsymbol{u}_{\diamond}^{n}-\boldsymbol{u}_{\diamond}^{n-1}\right\|_{L_{1}^{2}(\Omega)^{2}}+\frac{1}{\beta}\left\|g^{n}-g^{n-1}\right\|_{L_{1}^{2}\left(\Gamma_{u}\right)}$.

Dividing the square of this inequality by $\tau_{n}$, summing over $n$ with $1 \leq n \leq N$, using estimates (27), (21) and the initial condition of problem (23) we obtain (25).

3) To prove estimate (26), we choose $\boldsymbol{v}=\operatorname{grad} p^{n}$ in the first equation of (20) and apply Cauchy-Schwarz inequality we obtain $\left|p^{n}\right|_{H_{1}^{1}(\Omega)} \leq\left\|\frac{\boldsymbol{u}^{n}-\boldsymbol{u}^{n-1}}{\tau_{n}}\right\|_{L_{1}^{2}(\Omega)^{2}}+\alpha\left\|\boldsymbol{u}^{n}\right\|_{L_{1}^{2}(\Omega)^{2}}+\left\|\boldsymbol{f}^{n}\right\|_{L_{1}^{2}(\Omega)^{2}}$.

Multiplying the square of this inequality by $\tau_{n}$, summing over $n$, using estimates (24) and (25), we obtain (26).

Remark 2. Let $\Pi_{\tau}$ denote the operator which associates with any continuous function $v \in[0, T]$ the constant function $\Pi_{\tau} v$ equal to $v\left(t_{n}\right)$ on each interval 
]$\left.t_{n-1}, t_{n}\right], 1 \leq n \leq N$. Then, estimate (24) is equivalent to the following

$$
\begin{aligned}
\sup _{0 \leq m \leq n} & \left\|\boldsymbol{u}^{m}\right\|_{L_{1}^{2}(\Omega)^{2}} \leq c\left(\left\|\boldsymbol{u}_{0}\right\|_{L_{1}^{2}(\Omega)^{2}}+\left\|\Pi_{\tau} f\right\|_{L^{2}\left(0, t_{n} ; L_{1}^{2}(\Omega)^{2}\right)}\right. \\
& \left.+\|g(., 0)\|_{L_{1}^{2}\left(\Gamma_{u}\right)}+\left\|\Pi_{\tau} p_{b}\right\|_{L^{2}\left(0, t_{n} ; H_{1}^{\frac{1}{2}}\left(\Gamma_{p}\right)\right)}+\left\|\Pi_{\tau} g\right\|_{L^{2}\left(0, t_{n} ; L_{1}^{2}\left(\Gamma_{u}\right)\right)}\right) .
\end{aligned}
$$

In order to state the a priori error estimate, we observe that the family $\left(\boldsymbol{e}^{n}\right)_{0 \leq n \leq N}$, with $\boldsymbol{e}^{n}=\boldsymbol{u}\left(., t^{n}\right)-\boldsymbol{u}^{n}$ satisfies $\boldsymbol{e}^{0}=0$ and also, by integrating $\partial_{t} \boldsymbol{u}$ between $t_{n-1}$ and $t_{n}$ and subtracting (20) from (6) at time $t_{n}$,

$$
\left\{\begin{array}{l}
\forall \boldsymbol{v} \in L_{1}^{2}(\Omega)^{2},\left(\boldsymbol{e}^{n}, \boldsymbol{v}\right)_{1}+\alpha \tau_{n}\left(\boldsymbol{e}^{n}, \boldsymbol{v}\right)_{1}=\left(\boldsymbol{e}^{n-1}, \boldsymbol{v}\right)_{1}+\tau_{n}\left(\epsilon^{n}, \boldsymbol{v}\right)_{1} \\
\forall q \in H_{1 \diamond}^{1}(\Omega), b\left(\boldsymbol{e}^{n}, q\right)=0 .
\end{array}\right.
$$

Where the consistency error $\epsilon^{n}$ is given by

$$
\epsilon^{n}=\frac{1}{\tau_{n}} \int_{t_{n-1}}^{t_{n}}\left(\partial_{t} \boldsymbol{u}\right)(s) d s-\left(\partial_{t} \boldsymbol{u}\right)\left(t^{n}\right) .
$$

We assume that the velocity $\boldsymbol{u}$ of problem (4-5-6) belongs to $H^{2}\left(0, T ; L_{1}^{2}(\Omega)^{2}\right)$, then we can conclude this section, by recalling the main results concerning the a priori estimates, which are proven in [7, Prop. 3.2 and Cor. 3.1], for $n, 1 \leq n \leq N$ :

(i) $\left\|\boldsymbol{e}^{n}\right\|_{L_{1}^{2}(\Omega)^{2}} \leq \frac{1}{\sqrt{3 \alpha}}|\tau|\|\boldsymbol{u}\|_{H^{2}\left(0, t_{n} ; L_{1}^{2}(\Omega)^{2}\right)}$.

(ii) $\left(\sum_{m=1}^{n} \tau_{m}\left\|\frac{\boldsymbol{e}^{m}-\boldsymbol{e}^{m-1}}{\tau_{m}}\right\|_{L_{1}^{2}(\Omega)^{2}}^{2}\right)^{\frac{1}{2}} \leq \frac{1}{\sqrt{3}}|\tau|\|\boldsymbol{u}\|_{H^{2}\left(0, t_{n} ; L_{1}^{2}(\Omega)^{2}\right)}$,

$\left.\left.\left(\sum_{m=1}^{n} \tau_{m} \mid p\left(., t_{m}\right)-p^{m}\right)\right|_{H_{1}^{1}(\Omega)} ^{2}\right)^{\frac{1}{2}} \leq \frac{1}{\sqrt{3}}|\tau|\|\boldsymbol{u}\|_{H^{2}\left(0, t_{n} ; L_{1}^{2}(\Omega)^{2}\right)}$.

\subsection{The time and space discrete problem}

We now describe the space discretization of problem (18-19-20). For each $n$, $0 \leq n \leq N$, let $\left(\mathcal{T}_{n h}\right)_{h}$ be a regular family of triangulations of $\Omega$ by closed triangles, in the usual sense that

- for each $h, \bar{\Omega}$ is the union of all elements of $\mathcal{T}_{n h}$,

- $\Gamma_{p}$ is the union of whole edges of elements of $\mathcal{T}_{n h}$,

- there exists a constant $\sigma>0$ independant of $h, n$ and $T$ such that, for all

$T$ in $\mathcal{T}_{n h}, \frac{h_{T}}{\rho_{T}} \leq \sigma$, where $h_{T}$ is the diameter of $T$, and $\rho_{T}$ the diameter of its inscribed circle,

- $h_{n}$ the maximum of the diameters of the elements of $\mathcal{T}_{n h}$,

- $\mathcal{E}_{n h}$ is the set of all edges $e$ of elements $T$ of $\mathcal{T}_{n h}$,

- $\mathcal{E}_{n h}^{0}$ is the subset of $\mathcal{E}_{n h}$ which elements are not contained in $\partial \Omega$, 
- $\mathcal{V}_{n h}$ : is the set of vertices of the elements of $\mathcal{T}_{n h}$,

- $\mathcal{V}_{n h}^{0}$ : is the subset of $\mathcal{V}_{n h}$ which elements are inside $\Omega$,

- $\mathcal{V}_{n h}^{b}=\mathcal{V}_{n h} \backslash \mathcal{V}_{n h}^{0}$ : is the subset of $\mathcal{V}_{n h}$ of boundary vertices.

For each triangle $T$ and nonnegative integer $k$, we denote by $P_{k}(T)$ the space of restrictions to $T$ of polynomials with degree $\leq k$. At each time step, the discrete space of velocities is:

$$
X_{n h}(\Omega)=\left\{\boldsymbol{v}_{h} \in L_{1}^{2}(\Omega)^{2} / \forall T \in \mathcal{T}_{n h},\left.\boldsymbol{v}_{h}\right|_{T} \in P_{0}(T)^{2}\right\},
$$

its interpolation operator is the orthogonal projection operator $\Pi_{n h}: L_{1}^{2}(\Omega)^{2} \rightarrow X_{n h}$ associated with the scalar product of $L_{1}^{2}(\Omega)^{2}$ and verify, for every $0 \leq s \leq 1$

$$
\forall \boldsymbol{v} \in H_{1}^{s}(\Omega), \quad\left\|\boldsymbol{v}-\Pi_{n h} \boldsymbol{v}\right\|_{L_{1}^{2}(\Omega)^{2}} \leq C h_{n}^{s}\|\boldsymbol{v}\|_{H_{1}^{s}(\Omega)^{2}} .
$$

We assume that the pressure is continuous whence the choice of discrete space as proposed in [1]:

$$
M_{n h}(\Omega)=\left\{q_{h} \in H_{1}^{1}(\Omega) / \forall T \in \mathcal{T}_{n h},\left.q_{h}\right|_{T} \in P_{1}(T)\right\},
$$

its degrees of freedom are defined at the nodes of $\mathcal{V}_{n h}$ and its interpolation operator $i_{n h}: H_{1}^{1}(\Omega) \rightarrow M_{n h}(\Omega)$ is the standard Lagrange interpolation operator at the nodes of $\mathcal{V}_{n h}$ with values in $M_{n h}$ and verify, for every $\frac{1}{2}<s \leq 1$

$$
\forall q \in H_{1}^{s+1}(\Omega),\left|q-i_{n h} q\right|_{H_{1}^{1}(\Omega)} \leq C h_{n}^{s}\|q\|_{H_{1}^{s+1}(\Omega)},
$$

finally to approximate functions with zero trace on $\Gamma_{p}$, we set

$$
M_{n h}^{0}(\Omega)=\left\{q_{h} \in M_{n h}(\Omega) / q_{h}=0 \text { on } \Gamma_{p}\right\} .
$$

\subsubsection{Variational formulation of the discrete problem}

For every data $\left(\boldsymbol{f}, p_{b}\right)$ which belongs to $C^{0}\left(0, T ; L_{1}^{2}(\Omega)^{2}\right) \times C^{0}\left(0, T ; H_{1}^{s+\frac{1}{2}}\left(\Gamma_{p}\right)\right)$, $s>\frac{1}{2}, g$ belongs to $C^{0}\left(0, T ; L_{1}^{2}\left(\Gamma_{u}\right)\right)$ and $\boldsymbol{u}_{0}$ belongs to $L_{1}^{2}(\Omega)^{2}$ satisfies

$\operatorname{div} \boldsymbol{u}_{0}=0$ in $\Omega$, the discret problem constructed by the Galerkin method from (18)-(19)-(20) reads:

Find $\left(\boldsymbol{u}_{h}^{n}\right)_{0 \leq n \leq N} \in\left(X_{n h}(\Omega)\right)^{N+1}$ and $\left(p_{h}^{n}\right)_{1 \leq n \leq N} \in\left(M_{n h}(\Omega)\right)^{N}$ such that

$$
\boldsymbol{u}_{h}^{0}=\Pi_{0 h} \boldsymbol{u}^{0} \quad \text { in } \Omega,
$$

$\forall n, 1 \leq n \leq N$

$$
p_{h}^{n}=i_{n h} p_{b} \quad \text { on } \quad \Gamma_{p},
$$

$$
\begin{array}{r}
\forall \boldsymbol{v}_{h} \in X_{n h}(\Omega),\left(\boldsymbol{u}_{h}^{n}, \boldsymbol{v}_{h}\right)_{1}+\alpha \tau_{n}\left(\boldsymbol{u}_{h}^{n}, \boldsymbol{v}_{h}\right)_{1}+\tau_{n} b\left(\boldsymbol{v}_{h}, p_{h}^{n}\right)=\left(\boldsymbol{u}_{h}^{n-1}, \boldsymbol{v}_{h}\right)_{1} \\
+\tau_{n} \int_{\Omega}\left(\boldsymbol{f}^{n} \cdot \boldsymbol{v}_{h}\right)(r, z) r d r d z,
\end{array}
$$

$\forall q_{h} \in M_{n h}^{0}(\Omega), b\left(\boldsymbol{u}_{h}^{n}, q_{h}\right)=\left\langle g^{n}, q_{h}\right\rangle_{\Gamma_{u}}$. 
The function $\boldsymbol{v}_{h}=\left.\left(\operatorname{grad} q_{h}\right)\right|_{T}$ belongs to $X_{n h}$ and we have the inf-sup condition

$$
\forall q_{h} \in M_{n h}(\Omega), \sup _{\boldsymbol{v}_{h} \in X_{n h}} \frac{b\left(\boldsymbol{v}_{h}, q_{h}\right)}{\left\|\boldsymbol{v}_{h}\right\|_{L_{1}^{2}(\Omega)^{2}}}=\left|q_{h}\right|_{H_{1}^{1}(\Omega)} .
$$

Let the discrete kernel

$$
\mathbb{V}_{n h}(\Omega)=\left\{\boldsymbol{v}_{h} \in X_{n h}(\Omega) ; \forall q_{h} \in M_{n h}^{0}(\Omega), b\left(\boldsymbol{v}_{h}, q_{h}\right)=0\right\} .
$$

The choice of the lifting operator $\mathcal{L}$, which is introduced in (9) yields that, if $p_{b}^{n}$ belongs to $H_{1}^{s+\frac{1}{2}}\left(\Gamma_{p}\right), \frac{1}{2}<s \leq 1$, then $\bar{p}_{b}^{n}$ belongs to $H_{1}^{s+1}(\Omega)$, therefore its interpolate by Lagrange interpolation operator $i_{n h}\left(\bar{p}_{b}^{n}\right)$ is well-defined. Define the linear operator $B: X_{n h} \rightarrow M_{n h}^{0}(\Omega)^{\prime}$ by

$$
\left\langle B_{n h} \boldsymbol{v}, q\right\rangle_{M_{n h}^{0}(\Omega)^{\prime} \times M_{n h}^{0}(\Omega)}=b(\boldsymbol{v}, q) .
$$

It follows from inf-sup condition (35) and [10, Chap. I, Lem. 4.1], that this operator is an isomorphism from $\mathbb{V}_{n h}(\Omega)^{\perp}$ onto $M_{n h}^{0}(\Omega)^{\prime}$.

Hence, for $g^{n} \in M_{n h}^{0}(\Omega)^{\prime}$ there exists a unique $\boldsymbol{u}_{b h}^{n} \in \mathbb{V}_{n h}(\Omega)^{\perp}$ such that $\forall q \in M_{n h}^{0}(\Omega), b\left(\boldsymbol{u}_{b h}^{n}, q\right)=\left\langle g^{n}, q\right\rangle_{\Gamma_{u}}$ and

$$
\beta\left\|\boldsymbol{u}_{b h}^{n}\right\|_{L_{1}^{2}(\Omega)^{2}} \leq\left\|g^{n}\right\|_{L_{1}^{2}\left(\Gamma_{u}\right)} .
$$

When setting $\boldsymbol{u}_{\diamond h}^{n}=\boldsymbol{u}_{h}^{n}-\boldsymbol{u}_{b h}^{n}$, thus, problem (32-33-34) is equivalent to find $\left(\boldsymbol{u}_{\diamond h}^{n}\right)_{0 \leq n \leq N} \in\left(\mathbb{V}_{n h}(\Omega)\right)^{N+1}$ such that

$$
\boldsymbol{u}_{\diamond h}^{0}=\boldsymbol{u}_{h}^{0}-\boldsymbol{u}_{b h}^{0}=\Pi_{0 h} \boldsymbol{u}^{0}-\boldsymbol{u}_{b h}^{0},
$$

and, for all $n, \forall 1 \leq n \leq N$,

$$
\begin{aligned}
& \forall \boldsymbol{v}_{h} \in \mathbb{V}_{n h}(\Omega),\left(\boldsymbol{u}_{\diamond h}^{n}, \boldsymbol{v}_{h}\right)_{1}-\left(\boldsymbol{u}_{\diamond h}^{n-1}, \boldsymbol{v}_{h}\right)_{1}+\alpha \tau_{n}\left(\boldsymbol{u}_{\diamond h}^{n}, \boldsymbol{v}_{h}\right)_{1}= \\
& \tau_{n}\left(\boldsymbol{f}^{n}, \boldsymbol{v}_{h}\right)_{1}-\tau_{n} b\left(\boldsymbol{v}_{h}, i_{n h}\left(\bar{p}_{b}^{n}\right)\right) .
\end{aligned}
$$

Theorem 4. For every data

$\left(\boldsymbol{f}, p_{b}\right)$ belongs to $C^{0}\left(0, T ; L_{1}^{2}(\Omega)^{2}\right) \times C^{0}\left(0, T ; H_{1}^{s+\frac{1}{2}}\left(\Gamma_{p}\right)\right), s>\frac{1}{2}$, $g$ belongs to $C^{0}\left(0, T ; L_{1}^{2}\left(\Gamma_{u}\right)\right)$ and $\boldsymbol{u}_{0}$ belongs to $L_{1}^{2}(\Omega)^{2}$ satisfies $\operatorname{div} \boldsymbol{u}_{0}=0$ in $\Omega$. Then problem (32-33-34) has a unique solution $\left(\boldsymbol{u}_{h}^{n}, p_{h}^{n}\right)$ such that

$$
\forall n, 0 \leq n \leq N, \boldsymbol{u}_{h}^{n} \in X_{n h}(\Omega), \quad \text { and } \quad \forall n, 1 \leq n \leq N, p_{h}^{n} \in M_{n h}(\Omega) .
$$

Moreover, $\left(\boldsymbol{u}_{h}^{n}\right)_{0 \leq n \leq N}$ satisfies

$$
\begin{gathered}
\left\|\boldsymbol{u}_{h}^{n}\right\|_{L_{1}^{2}(\Omega)^{2}} \leq\left\|\boldsymbol{u}_{0}\right\|_{L_{1}^{2}(\Omega)^{2}}+\sqrt{\frac{2}{\alpha}}\left(\sum_{m=1}^{n} \tau_{m}\left(\left\|\boldsymbol{f}^{m}\right\|_{L_{1}^{2}(\Omega)^{2}}^{2}+c_{0}^{2}\left\|p_{b}^{m}\right\|_{H_{1}^{s+\frac{1}{2}}\left(\Gamma_{p}\right)}^{2}\right)\right)^{\frac{1}{2}} \\
+\|g(., 0)\|_{L_{1}^{2}\left(\Gamma_{u}\right)}+\left\|g^{n}\right\|_{L_{1}^{2}\left(\Gamma_{u}\right)} .
\end{gathered}
$$

Proof. Applying estimate (24) to problem (32-33-34) and using the fact that $\left\|\Pi_{0 h} \boldsymbol{u}^{0}\right\|_{L_{1}^{2}(\Omega)^{2}} \leq\left\|\boldsymbol{u}^{0}\right\|_{L_{1}^{2}(\Omega)^{2}}$, we obtain the desired estimate. 


\subsection{A priori error estimates}

To establish error estimates, we insert in the error equation an arbitrary element $q_{h}^{n} \in M_{n h}(\Omega)$ and we obtain

$\forall \boldsymbol{v}_{h} \in X_{n h} ;\left(\Pi_{n h} \boldsymbol{u}^{n}-\boldsymbol{u}_{h}^{n}, \boldsymbol{v}_{h}\right)_{1}+\alpha \tau_{n}\left(\Pi_{n h} \boldsymbol{u}^{n}-\boldsymbol{u}_{h}^{n}, \boldsymbol{v}_{h}\right)_{1}+\tau_{n} b\left(\boldsymbol{v}_{h}, q_{h}^{n}-p_{h}^{n}\right)$

$$
=\left(\boldsymbol{u}^{n-1}-\mathbf{u}_{h}^{n-1}, \boldsymbol{v}_{h}\right)_{1}-\tau_{n} b\left(\boldsymbol{v}_{h}, p^{n}-q_{h}^{n}\right),
$$

with the starting value $\Pi_{0 h} \boldsymbol{u}^{0}-\boldsymbol{u}_{h}^{0}=0$ in $\Omega$.

Proposition 1. We suppose that $\boldsymbol{u}_{0} \in H_{1}^{s}(\Omega)^{2}$ and the solution $\left(\boldsymbol{u}^{n}, p^{n}\right) \in H_{1}^{s}(\Omega)^{2} \times H_{1}^{s+1}(\Omega)^{2}, \frac{1}{2}<s \leq 1$. Then for all $n, 1 \leq n \leq N$

$$
\begin{aligned}
\left\|\boldsymbol{u}^{n}-\boldsymbol{u}_{h}^{n}\right\|_{L_{1}^{2}(\Omega)^{2}} \leq c\left(\left(\sum_{m=1}^{n} \tau_{m}\left(h_{m}\right)^{2 s}\right.\right. & \left.\left\|p^{m}\right\|_{H_{1}^{s+1}(\Omega)}^{2}\right)^{\frac{1}{2}} \\
& \left.+\sum_{m=0}^{n}\left(h_{m}\right)^{s}\left\|\boldsymbol{u}^{m}\right\|_{H_{1}^{s}(\Omega)^{2}}\right) .
\end{aligned}
$$

Proof. In the error equation (39), we choose $q_{h}^{n}=i_{n h}\left(p^{n}\right)$ then, $p_{h}^{n}-q_{h}^{n}$ belongs to $M_{n h}^{0}(\Omega)$, and for any $\boldsymbol{v}_{h} \in \mathbb{V}_{n h}(\Omega)$,

$$
\begin{aligned}
\left(\Pi_{n h} \boldsymbol{u}^{n}-\boldsymbol{u}_{h}^{n}, \boldsymbol{v}_{h}\right)_{1}+\alpha \tau_{n}\left(\Pi_{n h} \boldsymbol{u}^{n}-\boldsymbol{u}_{h}^{n}, \boldsymbol{v}_{h}\right)_{1}=\left(\boldsymbol{u}^{n-1}-\boldsymbol{u}_{h}^{n-1}, \boldsymbol{v}_{h}\right)_{1} & \\
& -\tau_{n} b\left(\boldsymbol{v}_{h}, p^{n}-q_{h}^{n}\right),
\end{aligned}
$$

we substract $\left(\Pi_{n-1, h} \boldsymbol{u}^{n-1}, \boldsymbol{v}_{h}\right)$ to this equation,we obtain for any $\boldsymbol{v}_{h} \in \mathbb{V}_{n h}(\Omega)$,

$$
\begin{array}{r}
\left(\left(\Pi_{n h} \boldsymbol{u}^{n}-\boldsymbol{u}_{h}^{n}\right)-\left(\Pi_{n-1, h} \boldsymbol{u}^{n-1}-\boldsymbol{u}_{h}^{n-1}\right), \boldsymbol{v}_{h}\right)_{1}+\alpha \tau_{n}\left(\Pi_{n h} \boldsymbol{u}^{n}-\boldsymbol{u}_{h}^{n}, \boldsymbol{v}_{h}\right)_{1} \\
=-\tau_{n} b\left(\boldsymbol{v}_{h}, p^{n}-q_{h}^{n}\right)+\left(\boldsymbol{u}^{n-1}-\Pi_{n-1, h} \boldsymbol{u}^{n-1}, \boldsymbol{v}_{h}\right)_{1} .
\end{array}
$$

Recall that $\Pi_{n h}$ is the orthogonal projection operator from $L_{1}^{2}(\Omega)^{2}$ onto $X_{n h}$, then $\left.\Pi_{n h} \boldsymbol{u}^{n}\right|_{T} \in P_{0}(T)^{2}$ and $\forall p \in P_{0}(T)^{2} \int_{T}\left(\Pi_{n h} \boldsymbol{u}^{n}-\boldsymbol{u}^{n}\right) p r d r d z=0$. For an arbitray $q_{h} \in M_{n h}^{0}(\Omega)$, we choose $p=\operatorname{grad} q_{h} \in P_{0}(T)^{2}$, then

$$
\forall q_{h} \in M_{n h}^{0}(\Omega), \int_{T}\left(\Pi_{n h} \boldsymbol{u}^{n}-\boldsymbol{u}^{n}\right) \operatorname{grad} q_{h} r d r d z=0
$$

therefore by using equations (20) with $q=q_{h} \in M_{n h}^{0}(\Omega) \subset H_{1 \diamond}^{1}(\Omega)$ and the second equation of (34) we obtain

$$
\begin{aligned}
\forall q_{h} \in M_{n h}^{0}(\Omega), \sum_{T \in \mathcal{T}_{n h}} \int_{T} \Pi_{n h} \boldsymbol{u}^{n} \operatorname{grad} q_{h} r d r d z & =\sum_{T \in \mathcal{T}_{n h}} \int_{T} \boldsymbol{u}^{n} \operatorname{grad} q_{h} r d r d z \\
& =\sum_{T \in \mathcal{T}_{n h}} \int_{T} \boldsymbol{u}_{h}^{n} \operatorname{grad} q_{h} r d r d z \\
& =\left\langle g^{n}, q_{h}\right\rangle_{\Gamma_{u}},
\end{aligned}
$$


then, $\Pi_{n h} \boldsymbol{u}^{n}-\boldsymbol{u}_{h}^{n} \in \mathbb{V}_{n h}(\Omega)$. Taking $\boldsymbol{v}_{h}=\Pi_{n h} \boldsymbol{u}^{n}-\boldsymbol{u}_{h}^{n}$ in (41), and apply Cauchy-Schwarz inequality we obtain

$$
\begin{gathered}
\left\|\Pi_{n h} \boldsymbol{u}^{n}-\boldsymbol{u}_{h}^{n}\right\|_{L_{1}^{2}(\Omega)^{2}} \leq\left\|\Pi_{n-1, h} \boldsymbol{u}^{n-1}-\boldsymbol{u}_{h}^{n-1}\right\|_{L_{1}^{2}(\Omega)^{2}}+\tau_{n}\left|p^{n}-q_{h}^{n}\right|_{H_{1}^{1}(\Omega)} \\
+\left\|\boldsymbol{u}^{n-1}-\Pi_{n-1, h} \boldsymbol{u}_{h}^{n-1}\right\|_{L_{1}^{2}(\Omega)^{2}} .
\end{gathered}
$$

Summing over $n$ yields

$$
\left\|\Pi_{n h} \boldsymbol{u}^{n}-\boldsymbol{u}_{h}^{n}\right\|_{L_{1}^{2}(\Omega)^{2}} \leq \sum_{m=1}^{n} \tau_{m}\left|p^{m}-q_{h}^{m}\right|_{H_{1}^{1}(\Omega)}+\sum_{m=1}^{n}\left\|\boldsymbol{u}^{m-1}-\Pi_{m-1, h} \boldsymbol{u}^{m-1}\right\|_{L_{1}^{2}(\Omega)^{2}} .
$$

Therefore by triangle inequality and the fact that $q_{h}^{m}=i_{m h}\left(p^{m}\right)$ we obtain $\left\|\boldsymbol{u}^{n}-\boldsymbol{u}_{h}^{n}\right\|_{L_{1}^{2}(\Omega)^{2}} \leq\left\|\boldsymbol{u}^{n}-\Pi_{n h} \boldsymbol{u}^{n}\right\|_{L_{1}^{2}(\Omega)^{2}}+\left\|\Pi_{n h} \boldsymbol{u}^{n}-\boldsymbol{u}_{h}^{n}\right\|_{L_{1}^{2}(\Omega)^{2}}$,

$$
\leq \sum_{m=1}^{n} \tau_{m}\left|p^{m}-i_{m h} p^{m}\right|_{H_{1}^{1}(\Omega)}+\sum_{m=0}^{n}\left\|\boldsymbol{u}^{m}-\Pi_{m h} \boldsymbol{u}^{m}\right\|_{L_{1}^{2}(\Omega)^{2}},
$$

Finaly, we apply the discrete Cauchy-Schwarz inequality, and use estimates (30) and (31) we obtain the desired a priori estimate.

Proposition 2. If the assumptions of Proposition 1 are satisfied, the following a priori error estimate holds for $n, 1 \leq n \leq N$,

$$
\begin{gathered}
\left\|\frac{1}{\tau_{n}} \Pi_{n h}\left(\left(\boldsymbol{u}^{n}-\boldsymbol{u}_{h}^{n}\right)-\left(\boldsymbol{u}^{n-1}-\boldsymbol{u}_{h}^{n-1}\right)\right)+\boldsymbol{g r a d}\left(p^{n}-p_{h}^{n}\right)\right\|_{L_{1}^{2}(\Omega)^{2}} \\
\leq c\left(\left(\sum_{m=1}^{n} \tau_{m}\left(h_{m}\right)^{2 s}\left\|p^{m}\right\|_{H_{1}^{s+1}(\Omega)}^{2}\right)^{\frac{1}{2}}+\sum_{m=0}^{n} \tau_{m}\left(h_{m}\right)^{s}\left\|\boldsymbol{u}^{m}\right\|_{H_{1}^{s}(\Omega)^{2}}\right) \\
+c\left(h_{n}\right)^{s}\left\|p^{n}\right\|_{H_{1}^{s+1}(\Omega)} .
\end{gathered}
$$

Proof. We can rewrite the error equation (39) as follows $\left(\frac{1}{\tau_{n}} \Pi_{n h}\left(\left(\boldsymbol{u}^{n}-\boldsymbol{u}_{h}^{n}\right)-\left(\boldsymbol{u}^{n-1}-\boldsymbol{u}_{h}^{n-1}\right)\right)+\operatorname{grad}\left(q_{h}^{n}-p_{h}^{n}\right), \boldsymbol{v}_{h}\right)_{1}$

$$
=-\alpha\left(\Pi_{n h}\left(\boldsymbol{u}^{n}-\boldsymbol{u}_{h}^{n}\right), \boldsymbol{v}_{h}\right)_{1}+b\left(\boldsymbol{v}_{h}, q_{h}^{n}-p^{n}\right) .
$$

We choose $\boldsymbol{v}_{h}=\frac{1}{\tau_{n}} \Pi_{n h}\left(\left(\boldsymbol{u}^{n}-\boldsymbol{u}_{h}^{n}\right)-\left(\boldsymbol{u}^{n-1}-\boldsymbol{u}_{h}^{n-1}\right)\right)+\operatorname{grad}\left(q_{h}^{n}-p_{h}^{n}\right)$, then

$$
\begin{aligned}
& \| \frac{1}{\tau_{n}} \Pi_{n h}\left(\left(\boldsymbol{u}^{n}-\boldsymbol{u}_{h}^{n}\right)-\left(\boldsymbol{u}^{n-1}-\right.\right.\left.\left.\boldsymbol{u}_{h}^{n-1}\right)\right)+\operatorname{grad}\left(q_{h}^{n}-p_{h}^{n}\right) \|_{L_{1}^{2}(\Omega)^{2}} \\
& \leq c\left(\left\|\boldsymbol{u}^{n}-\boldsymbol{u}_{h}^{n}\right\|_{L_{1}^{2}(\Omega)^{2}}+\left|q_{h}^{n}-p^{n}\right|_{H_{1}^{1}(\Omega)}\right) .
\end{aligned}
$$

On the other hand, by triangle inequality we have

$$
\begin{aligned}
& \left\|\frac{1}{\tau_{n}} \Pi_{n h}\left(\left(\boldsymbol{u}^{n}-\boldsymbol{u}_{h}^{n}\right)-\left(\boldsymbol{u}^{n-1}-\boldsymbol{u}_{h}^{n-1}\right)\right)+\operatorname{grad}\left(p^{n}-p_{h}^{n}\right)\right\|_{L_{1}^{2}(\Omega)^{2}} \\
& \leq\left\|\frac{1}{\tau_{n}} \Pi_{n h}\left(\left(\boldsymbol{u}^{n}-\boldsymbol{u}_{h}^{n}\right)-\left(\boldsymbol{u}^{n-1}-\boldsymbol{u}_{h}^{n-1}\right)\right)+\operatorname{grad}\left(q_{h}^{n}-p_{h}^{n}\right)\right\|_{L_{1}^{2}(\Omega)^{2}}+\left|q_{h}^{n}-p^{n}\right|_{H_{1}^{1}(\Omega)} .
\end{aligned}
$$

Finaly by using estimates $(43),(40)$ and the approximate properties of $M_{n h}(\Omega)$, we obtain the a priori estimate (42). 


\section{A posteriori analysis}

For the time discretization and the space discretization, we describe a family of error indicators and prove upper and lower bounds for the error.

\subsection{The time discretisation}

For each $n, 1 \leq n \leq N$, we define the time error indicator, see [4] and [13]

$$
\eta_{n}=\left(\frac{\tau_{n}}{3}\right)^{\frac{1}{2}}\left\|\boldsymbol{u}_{h}^{n}-\boldsymbol{u}_{h}^{n-1}\right\|_{L_{1}^{2}(\Omega)^{2}} .
$$

Let $\boldsymbol{u}_{\tau}$ denote the function which is continous, affine on each interval $\left[t_{n-1}, t_{n}\right]$, $1 \leq n \leq N$, such that

$$
\forall n, 0 \leq n \leq N, \quad \boldsymbol{u}_{\tau}\left(t_{n}\right)=\boldsymbol{u}^{n},
$$

and $p_{\tau}$ denote the piecewise constant function such that

$$
\left.\forall n, 1 \leq n \leq N, \quad \forall t \in] t_{n-1}, t_{n}\right], \quad p_{\tau}(t)=p\left(t_{n}\right) .
$$

Then for all $\mathrm{t}$ in $\left.] t_{n-1}, t_{n}\right]$, the residual equation in variational form reads $\forall \boldsymbol{v} \in L_{1}^{2}(\Omega)^{2} ;\left(\partial_{t}\left(\boldsymbol{u}-\boldsymbol{u}_{\tau}\right), \boldsymbol{v}\right)_{1}+\alpha\left(\boldsymbol{u}-\boldsymbol{u}_{\tau}, \boldsymbol{v}\right)_{1}+b\left(\boldsymbol{v}, p_{*}-\Pi_{\tau} p_{*}\right)=\left(\boldsymbol{f}-\Pi_{\tau} \boldsymbol{f}, \boldsymbol{v}\right)_{1}$

$$
-\alpha\left(\boldsymbol{u}_{\tau}-\boldsymbol{u}^{n}, \boldsymbol{v}\right)_{1}-b\left(\boldsymbol{v}, \bar{p}_{b}-\Pi_{\tau} \bar{p}_{b}\right)
$$

$$
\forall q \in H_{1 \diamond}^{1}(\Omega) ; b\left(\boldsymbol{u}-\boldsymbol{u}_{\tau}, q\right)=\left\langle g-\Pi_{\tau} g, q\right\rangle_{\Gamma_{u}},
$$

where $\Pi_{\tau}$ is introduced in Remark 2 by

$$
\left.\forall t \in] t_{n-1}, t_{n}\right], \quad\left(\Pi_{\tau} \boldsymbol{v}\right)(t)=\boldsymbol{v}\left(t_{n}\right),
$$

and we recall that $p=p_{*}+\bar{p}_{b}$. Let the regularity parameter

$$
\sigma_{\tau}=\max _{1 \leq n \leq N} \frac{\tau_{n}}{\tau_{n-1}}
$$

where $\tau_{0}=\tau_{1}$.

\subsubsection{The reliability of the indicator}

Proposition 3. The following a posteriori error estimate holds, for $1 \leq n \leq N$,

$$
\begin{gathered}
\left\|\boldsymbol{u}\left(., t_{n}\right)-\boldsymbol{u}^{n}\right\|_{L_{1}^{2}(\Omega)^{2}} \leq \sqrt{2 \alpha}\left(\sum_{m=1}^{n} \eta_{m}^{2}\right)^{\frac{1}{2}} \\
+\sqrt{\frac{1}{\alpha}}\left(\left\|\boldsymbol{f}-\Pi_{\tau} \boldsymbol{f}\right\|_{L^{2}\left(0, t_{n} ; L_{1}^{2}(\Omega)^{2}\right)}+c_{0}\left\|p_{b}-\Pi_{\tau} p_{b}\right\|_{L^{2}\left(0, t_{n} ; H_{1}^{\frac{1}{2}}\left(\Gamma_{p}\right)\right)}\right) \\
+\sqrt{\alpha}\left(1+\sigma_{\tau}^{\frac{1}{2}}\right)\left(\sum_{m=0}^{n} \tau_{m}\left\|\boldsymbol{u}^{m}-\boldsymbol{u}_{h}^{m}\right\|_{L_{1}^{2}(\Omega)^{2}}^{2}\right)^{\frac{1}{2}}
\end{gathered}
$$


Proof. Applying estimate (16) to problem (45-46) at time $t=t_{n}$ and using the fact that $\left(\boldsymbol{u}-\boldsymbol{u}_{\tau}\right)(0)=0,\left(g-\Pi_{\tau} g\right)(0)=0,\left(g-\Pi_{\tau} g\right)\left(., t_{n}\right)=0$ and $\boldsymbol{u}^{n}=\boldsymbol{u}_{\tau}\left(t_{n}\right)=\Pi_{\tau} \boldsymbol{u}_{\tau}(t)$ we obtain $\left\|\boldsymbol{u}\left(., t_{n}\right)-\boldsymbol{u}^{n}\right\|_{L_{1}^{2}(\Omega)^{2}}^{2} \leq \alpha\left\|\left(\boldsymbol{u}_{\tau}-\Pi_{\tau} \boldsymbol{u}_{\tau}\right)\right\|_{L^{2}\left(0, t_{n} ; L_{1}^{2}(\Omega)^{2}\right)}^{2}$

$$
+\frac{1}{\alpha}\left(\left\|\boldsymbol{f}-\Pi_{\tau} f\right\|_{L^{2}\left(0, t_{n} ; L_{1}^{2}(\Omega)^{2}\right)}^{2}+\left\|\bar{p}_{b}-\Pi_{\tau} \bar{p}_{b}\right\|_{L^{2}\left(0, t_{n} ; H_{1}^{1}(\Omega)\right.}^{2}\right) .
$$

To estimate the first term in the right-hand side, we observe that on the interval ]$\left.t_{n-1}, t_{n}\right],\left(\boldsymbol{u}_{\tau}-\Pi_{\tau} \boldsymbol{u}_{\tau}\right)(t)=-\frac{t_{n}-t}{\tau_{n}}\left(\boldsymbol{u}^{n}-\boldsymbol{u}^{n-1}\right)$, thus by integrating this equation between $t_{n-1}$ and $t_{n}$ and using the fact that $\tau_{n}=t_{n}-t_{n-1}$, we obtain

$$
\left\|\boldsymbol{u}_{\tau}-\Pi_{\tau} \boldsymbol{u}_{\tau}\right\|_{L^{2}\left(t_{n-1}, t_{n} ; L_{1}^{2}(\Omega)^{2}\right)}^{2}=\left\|\boldsymbol{u}^{n}-\boldsymbol{u}^{n-1}\right\|_{L_{1}^{2}(\Omega)^{2}}^{2} \int_{t_{n-1}}^{t_{n}}\left(\frac{t_{n}-t}{\tau_{n}}\right)^{2} d t
$$

thus

$$
\left\|\boldsymbol{u}_{\tau}-\Pi_{\tau} \boldsymbol{u}_{\tau}\right\|_{L^{2}\left(t_{n-1}, t_{n} ; L_{1}^{2}(\Omega)^{2}\right)}=\left(\frac{\tau_{n}}{3}\right)^{\frac{1}{2}}\left\|\boldsymbol{u}^{n}-\boldsymbol{u}^{n-1}\right\|_{L_{1}^{2}(\Omega)^{2}} .
$$

On the other hand, triangle inequality yields $\left\|\boldsymbol{u}^{n}-\boldsymbol{u}^{n-1}\right\|_{L_{1}^{2}(\Omega)^{2}} \leq\left\|\boldsymbol{u}^{n}-\boldsymbol{u}_{h}^{n}\right\|_{L_{1}^{2}(\Omega)^{2}}+\left\|\boldsymbol{u}_{h}^{n}-\boldsymbol{u}_{h}^{n-1}\right\|_{L_{1}^{2}(\Omega)^{2}}+\left\|\boldsymbol{u}_{h}^{n-1}-\boldsymbol{u}^{n-1}\right\|_{L_{1}^{2}(\Omega)^{2}}$. Multipliying by $\left(\frac{\tau_{n}}{3}\right)^{\frac{1}{2}}$ and using the expression of the error indicator (44) gives $\left(\frac{\tau_{n}}{3}\right)^{\frac{1}{2}}\left\|\boldsymbol{u}^{n}-\boldsymbol{u}^{n-1}\right\|_{L_{1}^{2}(\Omega)^{2}} \leq\left(\frac{\tau_{n}}{3}\right)^{\frac{1}{2}}\left\|\boldsymbol{u}^{n}-\boldsymbol{u}_{h}^{n}\right\|_{L_{1}^{2}(\Omega)^{2}}+\eta_{n}$

$$
+\left(\frac{\tau_{n}}{3}\right)^{\frac{1}{2}}\left\|\boldsymbol{u}_{h}^{n-1}-\boldsymbol{u}^{n-1}\right\|_{L_{1}^{2}(\Omega)^{2}}
$$

The expression of $\sigma_{\tau}$ yields $\frac{\left(\frac{\tau_{n}}{3}\right)}{\left(\frac{\tau_{n-1}}{3}\right)} \leq \sigma_{\tau}$, thus $\left(\frac{\tau_{n}}{3}\right)^{\frac{1}{2}} \leq\left(\frac{\tau_{n-1}}{3}\right)^{\frac{1}{2}} \cdot\left(\sigma_{\tau}\right)^{\frac{1}{2}}$. Therefore by substituting the previous inequality and (50) in (49) we obtain

$$
\begin{aligned}
\left\|\boldsymbol{u}_{\tau}-\Pi_{\tau} \boldsymbol{u}_{\tau}\right\|_{L^{2}\left(t_{n-1}, t_{n} ; L_{1}^{2}(\Omega)^{2}\right)} \leq\left(\frac{\tau_{n}}{3}\right)^{\frac{1}{2}}\left\|\boldsymbol{u}^{n}-\boldsymbol{u}_{h}^{n}\right\|_{L_{1}^{2}(\Omega)^{2}}+\eta_{n} \\
+\left(\frac{\tau_{n-1}}{3}\right)^{\frac{1}{2}} \cdot\left(\sigma_{\tau}\right)^{\frac{1}{2}}\left\|\boldsymbol{u}_{h}^{n-1}-\boldsymbol{u}^{n-1}\right\|_{L_{1}^{2}(\Omega)^{2}},
\end{aligned}
$$

Summing over $n$ with $1 \leq n \leq N$, the square of this inequality we obtain $\left\|\boldsymbol{u}_{\tau}-\Pi_{\tau} \boldsymbol{u}_{\tau}\right\|_{L^{2}\left(0, t_{n} ; L_{1}^{2}(\Omega)^{2}\right)}^{2}$

$$
\leq 2 \sum_{m=1}^{n} \eta_{m}^{2}+\left(1+\sigma_{\tau}\right) \sum_{m=0}^{n} \tau_{m}\left\|\boldsymbol{u}^{m}-\boldsymbol{u}_{h}^{m}\right\|_{L_{1}^{2}(\Omega)^{2}}^{2} .
$$

Finaly by substituting (51) in (48) and using estimate (10) we obtain the desired a posteriori error estimate. 
Proposition 4. The following a posteriori error estimate holds, for $1 \leq n \leq N$,

$$
\begin{aligned}
& \left\|\partial_{t}\left(\boldsymbol{u}-\boldsymbol{u}_{\tau}\right)\right\|_{L^{2}\left(0, t_{n} ; L_{1}^{2}(\Omega)^{2}\right)} \leq c\left(\left\|\boldsymbol{f}-\Pi_{\tau} \boldsymbol{f}\right\|_{L^{2}\left(0, t_{n} ; L_{1}^{2}(\Omega)^{2}\right)}+\left(\sum_{m=1}^{n} \eta_{m}^{2}\right)^{\frac{1}{2}}\right. \\
& \left.+c_{0}\left\|p_{b}-\Pi_{\tau} p_{b}\right\|_{L^{2}\left(0, t_{n} ; H_{1}^{\frac{1}{2}}\left(\Gamma_{p}\right)\right)}+\left(1+\sqrt{\sigma_{\tau}}\right)\left(\sum_{m=0}^{n} \tau_{m}\left\|\boldsymbol{u}^{m}-\boldsymbol{u}_{h}^{m}\right\|_{L_{1}^{2}(\Omega)^{2}}^{2}\right)^{\frac{1}{2}}\right) .
\end{aligned}
$$

Proof. We take $\boldsymbol{v}$ equal to $\partial_{t}\left(\boldsymbol{u}-\boldsymbol{u}_{\tau}\right)$ in (45) and apply Cauchy-Schwarz inequality we obtain

$$
\begin{aligned}
\frac{1}{2}\left\|\partial_{t}\left(\boldsymbol{u}-\boldsymbol{u}_{\tau}\right)\right\|_{L_{1}^{2}(\Omega)^{2}}^{2}+ & \frac{\alpha}{2} \frac{d}{d t}\left\|\boldsymbol{u}-\boldsymbol{u}_{\tau}\right\|_{L_{1}^{2}(\Omega)^{2}}^{2} \leq\left\|\boldsymbol{f}-\Pi_{\tau} \boldsymbol{f}\right\|_{L_{1}^{2}(\Omega)^{2}}^{2} \\
& +\alpha^{2}\left\|\boldsymbol{u}_{\tau}-\Pi_{\tau} \boldsymbol{u}_{\tau}\right\|_{L_{1}^{2}(\Omega)^{2}}^{2}+\left\|\bar{p}_{b}-\Pi_{\tau} \bar{p}_{b}\right\|_{H_{1}^{1}(\Omega)}^{2} .
\end{aligned}
$$

Integrating between 0 and $t_{n}$, using estimate (10) and the fact that $\left(\boldsymbol{u}-\boldsymbol{u}_{\tau}\right)(0)=0$, we obtain

$$
\begin{array}{r}
\left\|\partial_{t}\left(\boldsymbol{u}-\boldsymbol{u}_{\tau}\right)\right\|_{L^{2}\left(0, t_{n} ; L_{1}^{2}(\Omega)^{2}\right)}^{2}+\alpha\left\|\left(\boldsymbol{u}-\boldsymbol{u}_{\tau}\right)\left(t_{n}\right)\right\|_{L_{1}^{2}(\Omega)^{2}}^{2} \leq 2\left(\left\|\boldsymbol{f}-\Pi_{\tau} \boldsymbol{f}\right\|_{L^{2}\left(0, t_{n} ; L_{1}^{2}(\Omega)^{2}\right)}^{2}\right. \\
\left.+\alpha^{2}\left\|\boldsymbol{u}_{\tau}-\Pi_{\tau} \boldsymbol{u}_{\tau}\right\|_{L^{2}\left(0, t_{n} ; L_{1}^{2}(\Omega)^{2}\right)}^{2}+c_{0}^{2}\left\|p_{b}-\Pi_{\tau} p_{b}\right\|_{L^{2}\left(0, t_{n} ; H_{1}^{\frac{1}{2}}\left(\Gamma_{p}\right)\right)}^{2}\right) .
\end{array}
$$

This last estimate and (51) implies (52).

Proposition 5. The following a posteriori error estimate holds, for $1 \leq n \leq N$ $\left\|p-p_{\tau}\right\|_{L^{2}\left(0, t_{n} ; H_{1}^{1}(\Omega)\right)} \leq c\left(\left\|\boldsymbol{f}-\Pi_{\tau} f\right\|_{L^{2}\left(0, t_{n} ; L_{1}^{2}(\Omega)^{2}\right)}\right.$

$$
\begin{gathered}
+c_{0}\left\|p_{b}-\Pi_{\tau} p_{b}\right\|_{L^{2}\left(0, t_{n} ; H_{1}^{\frac{1}{2}}\left(\Gamma_{p}\right)\right)} \\
\left.+\left(\sum_{m=1}^{n} \eta_{m}^{2}\right)^{\frac{1}{2}}+\left(1+\sqrt{\sigma_{\tau}}\right)\left(\sum_{m=0}^{n} \tau_{m}\left\|\boldsymbol{u}^{m}-\boldsymbol{u}_{h}^{m}\right\|_{L_{1}^{2}(\Omega)^{2}}^{2}\right)^{\frac{1}{2}}\right) .
\end{gathered}
$$

Proof. From equation (45) we have for all $\boldsymbol{v} \in L_{1}^{2}(\Omega)^{2}$,

$$
\begin{array}{r}
b\left(\boldsymbol{v}, p_{*}-\Pi_{\tau} p_{*}\right)=\left(\partial_{t}\left(\boldsymbol{u}_{\tau}-\boldsymbol{u}\right), \boldsymbol{v}\right)_{1}+\alpha\left(\boldsymbol{u}_{\tau}-\boldsymbol{u}, \boldsymbol{v}\right)_{1}+b\left(\boldsymbol{v}, \Pi_{\tau} \bar{p}_{b}-\bar{p}_{b}\right) \\
+\left(\boldsymbol{f}-\Pi_{\tau} \boldsymbol{f}, \boldsymbol{v}\right)_{1}+\alpha\left(\boldsymbol{u}^{n}-\boldsymbol{u}_{\tau}, \boldsymbol{v}\right)_{1} .
\end{array}
$$

Cauchy-Schwarz inequality and the inf-sup condition (7) yields

$$
\begin{gathered}
\beta\left\|p_{*}-\Pi_{\tau} p_{*}\right\|_{H_{1}^{1}(\Omega)} \leq\left\|\partial_{t}\left(\boldsymbol{u}_{\tau}-\boldsymbol{u}\right)\right\|_{L_{1}^{2}(\Omega)^{2}}+\alpha\left\|\boldsymbol{u}_{\tau}-\boldsymbol{u}\right\|_{L_{1}^{2}(\Omega)^{2}}+\left\|\bar{p}_{b}-\Pi_{\tau} \bar{p}_{b}\right\|_{H_{1}^{1}(\Omega)} \\
+\left\|\boldsymbol{f}-\Pi_{\tau} \boldsymbol{f}\right\|_{L_{1}^{2}(\Omega)^{2}}+\alpha\left\|\boldsymbol{u}^{n}-\boldsymbol{u}_{\tau}\right\|_{L_{1}^{2}(\Omega)^{2}} .
\end{gathered}
$$

Integrating between 0 and $t_{n}$ and the fact that $\boldsymbol{u}^{n}=\Pi_{\tau} \boldsymbol{u}_{\tau}$ we obtain

$$
\begin{aligned}
& \left\|p_{*}-\Pi_{\tau} p_{*}\right\|_{L^{2}\left(0, t_{n} ; H_{1}^{1}(\Omega)\right)}^{2} \\
& \quad \leq c\left(\left\|\partial_{t}\left(\boldsymbol{u}_{\tau}-\boldsymbol{u}\right)\right\|_{L^{2}\left(0, t_{n} ; L_{1}^{2}(\Omega)^{2}\right)}^{2}+c_{0}^{2}\left\|p_{b}-\Pi_{\tau} p_{b}\right\|_{L^{2}\left(0, t_{n} ; H_{1}^{\frac{1}{2}}\left(\Gamma_{p}\right)\right)}^{2}\right. \\
& \left.\quad+\left\|\boldsymbol{f}-\Pi_{\tau} \boldsymbol{f}\right\|_{L^{2}\left(0, t_{n} ; L_{1}^{2}(\Omega)^{2}\right)}^{2}+\alpha^{2}\left\|\Pi_{\tau} \boldsymbol{u}_{\tau}-\boldsymbol{u}_{\tau}\right\|_{L^{2}\left(0, t_{n} ; L_{1}^{2}(\Omega)^{2}\right)}^{2}\right) .
\end{aligned}
$$

Finaly by substituting (51) and (52) in the last inequality, using the fact that $p-p_{\tau}=\left(p_{*}-\Pi_{\tau} p_{*}\right)+\left(\bar{p}_{b}-\Pi_{\tau} \bar{p}_{b}\right)$ and triangle inequality we obtain the desired a posteriori error estimate. 
Corollary 2. The folowing a posteriori error estimate holds for $1 \leq n \leq N$, $\left\|\partial_{t}\left(\boldsymbol{u}-\boldsymbol{u}_{\tau}\right)\right\|_{L^{2}\left(0, t_{n} ; L_{1}^{2}(\Omega)^{2}\right)}+\left\|p-p_{\tau}\right\|_{L^{2}\left(0, t_{n} ; H_{1}^{1}(\Omega)\right)}$

$$
\begin{aligned}
& \leq c\left(\left\|\boldsymbol{f}-\Pi_{\tau} \boldsymbol{f}\right\|_{L^{2}\left(0, t_{n} ; L_{1}^{2}(\Omega)^{2}\right)}+\left\|p_{b}-\Pi_{\tau} p_{b}\right\|_{L^{2}\left(0, t_{n} ; H_{1}^{\frac{1}{2}}\left(\Gamma_{p}\right)\right)}\right. \\
& \left.+\left(\sum_{m=1}^{n} \eta_{m}^{2}\right)^{\frac{1}{2}}+\left(1+\sqrt{\sigma_{\tau}}\right)\left(\sum_{m=0}^{n} \tau_{m}\left\|\boldsymbol{u}^{m}-\boldsymbol{u}_{h}^{m}\right\|_{L_{1}^{2}(\Omega)^{2}}^{2}\right)^{\frac{1}{2}}\right) .
\end{aligned}
$$

\subsubsection{The efficiency of the indicator}

Proposition 6. Each indicator $\eta_{n}, 1 \leq n \leq N$, defined in (44) satisfies the following bound

$$
\begin{aligned}
\eta_{n} & \leq c\left(\left\|\boldsymbol{u}-\boldsymbol{u}_{\tau}\right\|_{H^{1}\left(t_{n-1}, t_{n} ; L_{1}^{2}(\Omega)^{2}\right)}+\left\|p-p_{\tau}\right\|_{L^{2}\left(t_{n-1}, t_{n} ; H_{1}^{1}(\Omega)\right)}\right. \\
& \left.+\left\|\boldsymbol{f}-\Pi_{\tau} \boldsymbol{f}\right\|_{L^{2}\left(t_{n-1}, t_{n} ; L_{1}^{2}(\Omega)^{2}\right)}+\left(1+\sqrt{\sigma_{\tau}}\right)\left(\sum_{m=n-1}^{n} \tau_{m}\left\|\boldsymbol{u}^{m}-\boldsymbol{u}_{h}^{m}\right\|_{L_{1}^{2}(\Omega)^{2}}^{2}\right)^{\frac{1}{2}}\right) .
\end{aligned}
$$

Moreover this estimate is local with respect to the time variable.

Proof. By the expression of the indicator $\eta_{n}$, triangle inequality, and the fact that $\left(\frac{\tau_{n}}{3}\right)^{\frac{1}{2}} \leq\left(\frac{\tau_{n-1}}{3}\right)^{\frac{1}{2}} \cdot\left(\sigma_{\tau}\right)^{\frac{1}{2}}$, we obtain

$$
\begin{gathered}
\eta_{n} \leq\left(\frac{\tau_{n}}{3}\right)^{\frac{1}{2}}\left\|\boldsymbol{u}_{h}^{n}-\boldsymbol{u}^{n}\right\|_{L_{1}^{2}(\Omega)^{2}}+\left(\frac{\tau_{n}}{3}\right)^{\frac{1}{2}}\left\|\boldsymbol{u}^{n}-\boldsymbol{u}^{n-1}\right\|_{L_{1}^{2}(\Omega)^{2}} \\
+\left(\frac{\tau_{n-1}}{3}\right)^{\frac{1}{2}}\left(\sigma_{\tau}\right)^{\frac{1}{2}}\left\|\boldsymbol{u}^{n-1}-\boldsymbol{u}_{h}^{n-1}\right\|_{L_{1}^{2}(\Omega)^{3}},
\end{gathered}
$$

then,

$$
\begin{aligned}
\eta_{n} \leq c\left(\left(\frac{\tau_{n}}{3}\right)^{\frac{1}{2}} \| \boldsymbol{u}^{n}\right. & -\boldsymbol{u}^{n-1} \|_{L_{1}^{2}(\Omega)^{2}} \\
& \left.+\left(1+\sqrt{\sigma_{\tau}}\right)\left(\sum_{m=n-1}^{n} \tau_{m}\left\|\boldsymbol{u}^{m}-\boldsymbol{u}_{h}^{m}\right\|_{L_{1}^{2}(\Omega)^{2}}^{2}\right)^{\frac{1}{2}}\right) .
\end{aligned}
$$

In order to evaluate the first term in the right-hand side, we take $\boldsymbol{v}=\boldsymbol{u}_{\tau}-\mathbf{u}^{n}$ in (45) and using the fact that $p_{*}+\bar{p}_{b}=p, \Pi_{\tau} p=p_{\tau}$ and $\boldsymbol{u}^{n}=\Pi_{\tau} \boldsymbol{u}_{\tau}$, we obtain $\left\|\boldsymbol{u}_{\tau}-\Pi_{\tau} \boldsymbol{u}_{\tau}\right\|_{L_{1}^{2}(\Omega)^{2}}^{2} \leq\left(\left\|\partial_{t}\left(\boldsymbol{u}_{\tau}-\boldsymbol{u}\right)\right\|_{L_{1}^{2}(\Omega)^{2}}+\alpha\left\|\boldsymbol{u}_{\tau}-\boldsymbol{u}\right\|_{L_{1}^{2}(\Omega)^{2}}\right.$

$$
\left.+\left\|p_{\tau}-p\right\|_{H_{1}^{1}(\Omega)}+\left\|\boldsymbol{f}-\Pi_{\tau} \boldsymbol{f}\right\|_{L_{1}^{2}(\Omega)^{2}}\right) \cdot\left\|\boldsymbol{u}_{\tau}-\Pi_{\tau} \boldsymbol{u}_{\tau}\right\|_{L_{1}^{2}(\Omega)^{2}} .
$$

Then,

$$
\begin{array}{r}
\left\|\boldsymbol{u}_{\tau}-\Pi_{\tau} \boldsymbol{u}_{\tau}\right\|_{L_{1}^{2}(\Omega)^{2}}^{2} \leq c\left(\left\|\partial_{t}\left(\boldsymbol{u}_{\tau}-\boldsymbol{u}\right)\right\|_{L_{1}^{2}(\Omega)^{2}}^{2}+\left\|\boldsymbol{u}_{\tau}-\boldsymbol{u}\right\|_{L_{1}^{2}(\Omega)^{2}}^{2}\right. \\
+\left\|p_{\tau}-p\right\|_{H_{1}^{1}(\Omega)}^{2}+\left\|\boldsymbol{f}-\Pi_{\tau} f\right\|_{L_{1}^{2}(\Omega)^{2}}^{2} .
\end{array}
$$

Integrating this inequality between $t_{n-1}$ and $t_{n}$ and using (49) we obtain

$$
\begin{aligned}
& \frac{\tau_{n}}{3}\left\|\boldsymbol{u}^{n}-\boldsymbol{u}^{n-1}\right\|_{L_{1}^{2}(\Omega)^{2}}^{2} \leq c\left(\left\|\boldsymbol{u}_{\tau}-\boldsymbol{u}\right\|_{H^{1}\left(t_{n-1}, t_{n} ; L_{1}^{2}(\Omega)^{2}\right)}^{2}\right. \\
& \left.\quad+\left\|p_{\tau}-p\right\|_{L^{2}\left(t_{n-1}, t_{n} ; H_{1}^{1}(\Omega)\right)}^{2}+\left\|\boldsymbol{f}-\Pi_{\tau} f\right\|_{L^{2}\left(t_{n-1}, t_{n} ; L_{1}^{2}(\Omega)^{2}\right)}^{2}\right) .
\end{aligned}
$$

Finally by substituting the previous inequality in (53) we obtain the desired estimate. 


\subsection{The space discretisation}

For each $T \in \mathcal{T}_{n h}$, we associate

- $\mathcal{E}_{T}$ the set of edges of $T$,

- $\mathcal{E}_{T}^{0}=\mathcal{E}_{T} \cap \mathcal{E}_{n h}^{0}$,

- $h_{e}$ the diameter of $e$

- $\mathcal{E}_{n h}^{\Gamma_{u}}=\left\{e \in \mathcal{E}_{n h} ; e \subset \Gamma_{u}\right\}$, where $\mathcal{E}_{n h}^{0}$ and $\mathcal{E}_{n h}$ are defined in section 3.2.

For each $n, 1 \leq n \leq N$ and each $T \in \mathcal{T}_{n h}$, we define the following error indicators

$$
\eta_{T}^{n}=\frac{1}{\tau_{n}}\left\|\boldsymbol{u}_{h}^{n-1}-\Pi_{n h} \boldsymbol{u}_{h}^{n-1}\right\|_{L_{1}^{2}(T)^{2}} \quad \text { and } \quad \eta_{\partial T}^{n}=\sum_{\mathcal{E}_{n h}^{\Gamma_{u}}} h_{e}^{\frac{1}{2}}\left\|\left[\boldsymbol{u}_{h}^{n} \cdot \boldsymbol{n}_{e}\right]_{e}\right\|_{L_{1}^{2}(e)},
$$

where the jumps $\left[\boldsymbol{u}_{h}^{n} \cdot \boldsymbol{n}_{e}\right]_{e}$ are constant on each $e$. Moreover, in the context of mesh adaptivity, the term $\boldsymbol{u}_{h}^{n-1}-\Pi_{n h} \boldsymbol{u}_{h}^{n-1}$ only differs from zero in the elements $T$ of $\mathcal{T}_{n h}$ that are the union of several elements of $\mathcal{T}_{n-1, h}$. Therefore these indicators can be computed readily and explicitly.

We approximate the boundary data $p_{b}^{n}$ by the Lagrange interpolation operator $i_{n h}$, with values in $M_{n h}^{0}(\Omega)$, i.e. for each continuous function $q$ belongs to $\Gamma_{p}, i_{n h} q$ is a piecewise affine function equal to $q$ on each node of $\mathcal{V}_{n h}^{b}$.

In order to prove the a posteriori estimates, we first write the residual equations. We recall that $p^{n}=p_{*}^{n}+\bar{p}_{b}^{n}$ and $p_{* h}^{n}=p_{h}^{n}-\mathcal{L}\left(i_{n h} p_{b}^{n}\right)$, where $\bar{p}_{b}^{n}=\mathcal{L}\left(p_{b}^{n}\right)$ and $p_{* h}^{n}$ is no longer a piecewise polynomial function.

Lemma 5. For any solutions $\left(\boldsymbol{u}^{n}, p^{n}\right)_{1 \leq n \leq N}$ of problem (18-19-20) and $\left(\boldsymbol{u}_{h}^{n}, p_{h}^{n}\right)_{1 \leq n \leq N}$ of problem (32-33-34), then $\left(\boldsymbol{u}^{n}-\boldsymbol{u}_{h}^{n}, p^{n}-p_{h}^{n}\right)_{1 \leq n \leq N}$ satisfies the residual equation

$\forall \boldsymbol{v} \in L_{1}^{2}(\Omega)^{2} ;\left(\boldsymbol{u}^{n}-\boldsymbol{u}_{h}^{n}, \boldsymbol{v}\right)_{1}+\alpha \tau_{n}\left(\boldsymbol{u}^{n}-\boldsymbol{u}_{h}^{n}, \boldsymbol{v}\right)_{1}+\tau_{n} b\left(\boldsymbol{v}, p_{*}^{n}-p_{* h}^{n}\right)$

$$
\begin{gathered}
=\left(\boldsymbol{u}^{n-1}-\boldsymbol{u}_{h}^{n-1}, v\right)_{1}+\left(\boldsymbol{u}_{h}^{n-1}-\Pi_{n h} \boldsymbol{u}_{h}^{n-1}, \boldsymbol{v}\right)_{1}+\tau_{n}\left(\boldsymbol{f}^{n}-\Pi_{n h} \boldsymbol{f}^{n}, \boldsymbol{v}\right)_{1} \\
-\tau_{n} b\left(\boldsymbol{v}, \mathcal{L}\left(p_{b}^{n}-i_{n h} p_{b}^{n}\right)\right),
\end{gathered}
$$

$\forall q \in H_{1 \diamond}^{1}(\Omega) ; b\left(\boldsymbol{u}^{n}-\boldsymbol{u}_{h}^{n}, q\right)=\left\langle g^{n}, q-q_{h}^{n}\right\rangle_{\Gamma_{u}}$

$$
-\frac{1}{2} \sum_{T \in \mathcal{T}_{n h}} \sum_{\mathcal{E}_{n h}^{\Gamma u}} \int_{e}\left[\boldsymbol{u}_{h}^{n} \cdot \boldsymbol{n}_{e}\right]_{e}(\tau)\left(q-q_{h}^{n}\right)(\tau) d \tau .
$$

Proof. Taking $\boldsymbol{v}_{h}$ equal to $\chi_{T} \mathbf{e}$ in (34), where $\chi_{T}$ is the characteristic function of $T$ and e runs through the canonical basis of base $\mathbb{R}^{2}$ we obtain $\forall T \in \mathcal{T}_{n h}$,

$\left(\boldsymbol{u}_{h}^{n}, \chi_{T} \mathbf{e}\right)_{1}+\alpha \tau_{n}\left(\boldsymbol{u}_{h}^{n}, \chi_{T} \mathbf{e}\right)_{1}+\tau_{n} b\left(\chi_{T} \mathbf{e}, p_{h}^{n}\right)=\left(\boldsymbol{u}_{h}^{n-1}, \chi_{T} \mathbf{e}\right)_{1}+\tau_{n}\left(\boldsymbol{f}^{n}, \chi_{T} \mathbf{e}\right)_{1}$.

Then,

$\boldsymbol{u}_{h}^{n}+\alpha \tau_{n} \boldsymbol{u}_{h}^{n}+\tau_{n} \operatorname{grad} p_{h}^{n}=\boldsymbol{u}_{h}^{n-1}+\frac{\tau_{n}}{\operatorname{meas}(T)} \int_{T} \boldsymbol{f}^{n} r d r d z=\Pi_{n h} \boldsymbol{u}_{h}^{n-1}+\tau_{n} \Pi_{n h} \boldsymbol{f}^{n}$.

Multiplying this equation by any $\boldsymbol{v} \in L_{1}^{2}(\Omega)^{2}$, integrating on each $T \in \mathcal{T}_{n h}$, and summing over all elements $T \in \mathcal{T}_{n h}$, we obtain

$$
\sum_{T \in \mathcal{T}_{n h}} \int_{T}\left(\boldsymbol{u}_{h}^{n}+\alpha \tau_{n} \boldsymbol{u}_{h}^{n}+\tau_{n} \operatorname{grad} p_{h}^{n}\right) \cdot \boldsymbol{v} r d r d z
$$




$$
=\sum_{T \in \mathcal{T}_{n h}} \int_{T}\left(\Pi_{n h} \boldsymbol{u}_{h}^{n-1}+\tau_{n} \Pi_{n h} \boldsymbol{f}^{n}\right) \cdot \boldsymbol{v} r d r d z,
$$

Finaly subtracting this equality from the first equation of (20) and using the fact that $p^{n}=p_{*}^{n}+\bar{p}_{b}^{n}$ where $\bar{p}_{b}^{n}=\mathcal{L}\left(p_{b}^{n}\right)$ and $p_{* h}^{n}=p_{h}^{n}-\mathcal{L}\left(i_{n h} p_{b}^{n}\right)$, we obtain the first equation of (54). On the other hand, the second equations of (20) and (34) gives $\forall q_{h}^{n} \in M_{n h}^{0}(\Omega), b\left(\boldsymbol{u}^{n}-\boldsymbol{u}_{h}^{n}, q_{h}^{n}\right)=0$. Then by Green's formula, we obtain $\forall q_{h}^{n} \in M_{n h}^{0}(\Omega)$,

$$
\begin{aligned}
b\left(\boldsymbol{u}^{n}-\boldsymbol{u}_{h}^{n}, q\right) & =b\left(\boldsymbol{u}^{n}-\boldsymbol{u}_{h}^{n}, q-q_{h}^{n}\right), \\
& =b\left(\boldsymbol{u}^{n}, q-q_{h}^{n}\right)-b\left(\boldsymbol{u}_{h}^{n}, q-q_{h}^{n}\right), \\
& =\left\langle g^{n}, q-q_{h}^{n}\right\rangle_{\Gamma_{u}}-\sum_{T \in \mathcal{T}_{n h}} \int_{T} \boldsymbol{u}_{h}^{n} \operatorname{grad}\left(q-q_{h}^{n}\right) r d r d z, \\
& =\left\langle g^{n}, q-q_{h}^{n}\right\rangle_{\Gamma_{u}}-\sum_{T \in \mathcal{T}_{n h}} \int_{\partial T \cap\left(\Omega \cup \Gamma_{u}\right)}\left(\boldsymbol{u}_{h}^{n} \cdot \boldsymbol{n}\right)(\tau)\left(q-q_{h}^{n}\right)(\tau) d \tau,
\end{aligned}
$$

whence the second line in (54).

\subsubsection{The reliability of the indicators}

Proposition 7. The following a posteriori error estimate holds between the solutions $\left(\boldsymbol{u}^{n}, p^{n}\right)_{1 \leq n \leq N}$ of problem (18-19-20) and $\left(\boldsymbol{u}_{h}^{n}, p_{h}^{n}\right)_{1 \leq n \leq N}$ of problem (32-33-34), for $n, 1 \leq n \leq N$,

$$
\begin{aligned}
\left\|\boldsymbol{u}^{n}-\boldsymbol{u}_{h}^{n}\right\|_{L_{1}^{2}(\Omega)^{2}}^{2} \leq & \left\|\boldsymbol{u}_{0}-\Pi_{0 h} \boldsymbol{u}_{0}\right\|_{L_{1}^{2}(\Omega)^{2}}^{2}+c\left(G_{n}^{2}+\sum_{T \in \mathcal{T}_{n h}}\left(\eta_{\partial T}^{n}\right)^{2}\right) \\
& +\frac{2}{\alpha} \sum_{m=1}^{n} \tau_{m}\left(J_{m}^{2}+\sum_{T \in \mathcal{T}_{n h}}\left(\eta_{T}^{m}\right)^{2}\right),
\end{aligned}
$$

where $J_{n}=\left\|f^{n}-\Pi_{n h} f^{n}\right\|_{L_{1}^{2}(\Omega)^{2}}, G_{n}=\left\|p_{b}^{n}-i_{n h} p_{b}^{n}\right\|_{H_{1}^{\frac{1}{2}}\left(\Gamma_{p}\right)}$.

Proof. To simplify, let $\boldsymbol{w}^{n}=\boldsymbol{u}^{n}-\boldsymbol{u}_{h}^{n}, r_{*}^{n}=p_{*}^{n}-p_{* h}^{n}$ and $\boldsymbol{F}^{n}=\boldsymbol{f}^{n}-\Pi_{n h} \boldsymbol{f}^{n}-\operatorname{grad} \mathcal{L}\left(p_{b}^{n}-i_{n h} p_{b}^{n}\right)+\frac{1}{\tau_{n}}\left(\boldsymbol{u}_{h}^{n-1}-\Pi_{n h} \boldsymbol{u}_{h}^{n-1}\right)$.

Therefore the residual equations (54) become

$$
\begin{gathered}
\forall \boldsymbol{v} \in L_{1}^{2}(\Omega)^{2},\left(\boldsymbol{w}^{n}, \boldsymbol{v}\right)_{1}+\alpha \tau_{n}\left(\boldsymbol{w}^{n}, \boldsymbol{v}\right)_{1}+\tau_{n} b\left(\boldsymbol{v}, r_{*}^{n}\right)=\left(\mathbf{w}^{n-1}, \boldsymbol{v}\right)_{1}+\tau_{n}\left(\boldsymbol{F}^{n}, \boldsymbol{v}\right)_{1}, \\
\forall q \in H_{1 \diamond}^{1}(\Omega), b\left(\boldsymbol{w}^{n}, q\right)=\left\langle g^{n}, q-R_{h}^{n} q\right\rangle_{\Gamma_{u}} \\
\quad-\frac{1}{2} \sum_{T \in \mathcal{T}_{n h}} \sum_{e \in \mathcal{E}_{n h}^{\Gamma}} \int_{e}\left[\boldsymbol{u}_{h}^{n} . \boldsymbol{n}_{e}\right]_{e}(\tau)\left(q-R_{h}^{n} q\right)(\tau) d \tau
\end{gathered}
$$

where $R_{h}^{n}$ denotes a Clément type regularization operator with values in $M_{n h}^{0}$ such as the Scott and Zhang operator [15]. This operator preserves the zero 
boundary trace and satisfies for each $T \in \mathcal{T}_{n h}$, and $e \in \mathcal{E}_{n h}^{\Gamma_{u}}$ see [8, Cor. IX.3.9], [15] and also [3] for the extension to weighted spaces,

$$
\forall q \in H_{1}^{1}(\Omega),\left\|q-R_{h}^{n} q\right\|_{L_{1}^{2}(e)} \leq \operatorname{che}_{e}^{\frac{1}{2}}\|q\|_{H_{1}^{1}\left(\Delta_{e}\right)},
$$

where $\Delta_{e}$ is an appropriate neighbourhood of $e$. Then from this inequality, there exists a unique $\mu^{n} \in H_{1 \diamond}^{1}(\Omega)$ such that

$$
\forall q \in H_{1 \diamond}^{1}(\Omega), \quad\left(\operatorname{grad} \mu^{n}, \operatorname{grad} q\right)_{1}=b\left(\boldsymbol{w}^{n}, q\right),
$$

and

$$
\left|\mu^{n}\right|_{H_{1}^{1}(\Omega)} \leq c\left(\sum_{T \in \mathcal{T}_{n h}} \sum_{e \in \mathcal{E}_{n h}^{\Gamma u}} h_{e}\left\|\left[\boldsymbol{u}_{h}^{n} \cdot \boldsymbol{n}_{e}\right]_{e}\right\|_{L_{1}^{2}(e)}^{2}\right)^{\frac{1}{2}} .
$$

Hence, $\boldsymbol{w}^{n}$ has the orthogonal decomposition: $\boldsymbol{w}^{n}=\boldsymbol{w}_{*}^{n}+\operatorname{grad} \mu^{n}$, with $\boldsymbol{w}_{*}^{n}$ belongs in $\mathbb{V}(\Omega)$. Taking $\boldsymbol{v}=\boldsymbol{w}_{*}^{n}$ in the first equation of problem (56), using the fact that $\left(\boldsymbol{w}^{n}, \boldsymbol{w}_{n}^{*}\right)_{1}=\left\|\boldsymbol{w}_{*}^{n}\right\|_{L_{1}^{2}(\Omega)^{2}}^{2},\left(\boldsymbol{w}^{n-1}, \boldsymbol{w}_{*}^{n}\right)_{1}=\left(\boldsymbol{w}_{*}^{n-1}, \boldsymbol{w}_{*}^{n}\right)_{1}$, and CauchySchwarz inequality we obtain

$\left\|\boldsymbol{w}_{*}^{n}\right\|_{L_{1}^{2}(\Omega)^{2}}^{2}-\left\|\boldsymbol{w}_{*}^{n-1}\right\|_{L_{1}^{2}(\Omega)^{2}}^{2}+\alpha \tau_{n}\left\|\boldsymbol{w}_{*}^{n}\right\|_{L_{1}^{2}(\Omega)^{2}}^{2} \leq \frac{\tau_{n}}{\alpha}\left\|\boldsymbol{F}^{n}\right\|_{L_{1}^{2}(\Omega)^{2}}^{2}$.

Then, $\left\|\boldsymbol{w}_{*}^{n}\right\|_{L_{1}^{2}(\Omega)^{2}}^{2}-\left\|\boldsymbol{w}_{*}^{n-1}\right\|_{L_{1}^{2}(\Omega)^{2}}^{2} \leq \frac{\tau_{n}}{\alpha}\left\|\boldsymbol{F}^{n}\right\|_{L_{1}^{2}(\Omega)^{2}}^{2}$.

Summig this inequality over $n$, yields

$$
\left\|\boldsymbol{w}_{*}^{n}\right\|_{L_{1}^{2}(\Omega)^{2}}^{2}-\left\|\boldsymbol{w}_{*}^{0}\right\|_{L_{1}^{2}(\Omega)^{2}}^{2} \leq \frac{1}{\alpha} \sum_{m=1}^{n} \tau_{m}\left\|\boldsymbol{F}^{m}\right\|_{L_{1}^{2}(\Omega)^{2}}^{2} .
$$

Using the fact that $\left\|\boldsymbol{w}^{n}\right\|_{L_{1}^{2}(\Omega)^{2}}^{2}=\left|\mu^{n}\right|_{H_{1}^{1}(\Omega)}^{2}+\left\|\boldsymbol{w}_{*}^{n}\right\|_{L_{1}^{2}(\Omega)^{2}}^{2}$, we obtain

$$
\left\|\boldsymbol{w}^{n}\right\|_{L_{1}^{2}(\Omega)^{2}}^{2} \leq\left\|\boldsymbol{w}^{0}\right\|_{L_{1}^{2}(\Omega)^{2}}^{2}+\left|\mu^{n}\right|_{H_{1}^{1}(\Omega)}^{2}+\frac{1}{\alpha} \sum_{m=1}^{n} \tau_{m}\left\|\boldsymbol{F}^{m}\right\|_{L_{1}^{2}(\Omega)^{2}}^{2} .
$$

Finaly by substituting (57) into this inequality, using the fact that

$$
\left\|\boldsymbol{F}^{n}\right\|_{L_{1}^{2}(\Omega)^{2}} \leq\left\|\mathbf{f}^{n}-\Pi_{n h} \mathbf{f}^{n}\right\|_{L_{1}^{2}(\Omega)^{2}}+\left\|p_{b}^{n}-i_{n h} p_{b}^{n}\right\|_{H_{1}^{\frac{1}{2}\left(\Gamma_{p}\right)}}+\eta_{T}^{n},
$$

and $\boldsymbol{w}^{n}=\boldsymbol{u}^{n}-\boldsymbol{u}_{h}^{n}$, we obtain the a posteriori estimate (55).

The next estimate is derived by similar arguments.

Proposition 8. The following a posteriori error estimate holds between the solutions $\left(\boldsymbol{u}^{n}, p^{n}\right)_{1 \leq n \leq N}$ of problem (18-19-20) and $\left(\boldsymbol{u}_{h}^{n}, p_{h}^{n}\right)_{1 \leq n \leq N}$ of problem (32-33-34), for $1 \leq n \leq N$,

$$
\begin{aligned}
& \sum_{m=1}^{n} \tau_{m}\left\|\frac{\left(\boldsymbol{u}^{m}-\boldsymbol{u}_{h}^{m}\right)-\left(\boldsymbol{u}^{m-1}-\boldsymbol{u}_{h}^{m-1}\right)}{\tau_{m}}+\boldsymbol{g r a d}\left(p^{m}-p_{h}^{m}\right)\right\|_{L_{1}^{2}(\Omega)^{2}}^{2} \\
& \leq \alpha\left\|\boldsymbol{u}^{0}-\boldsymbol{u}_{h}^{0}\right\|_{L_{1}^{2}(\Omega)^{2}}^{2}+\sum_{m=1}^{n} \tau_{m}\left(J_{m}^{2}+G_{m}^{2}+\sum_{T \in \tau_{m h}}\left(\left(\eta_{T}^{m} 2\right)+\left(\eta_{\partial T}^{m}\right)^{2}\right)\right) .
\end{aligned}
$$




\subsubsection{The efficiency of the indicators}

We will prove an upper bound for the error indicators. For each $T \in \mathcal{T}_{n h}$, let $\omega_{T}$ denote the union of triangles in $\mathcal{T}_{n h}$ that share at least an edge with $T$.

Proposition 9. For each $n, 1 \leq n \leq N$ and $T \in \mathcal{T}_{n h}$,

$\eta_{T}^{n} \leq \alpha\left\|\boldsymbol{u}^{n}-\boldsymbol{u}_{h}^{n}\right\|_{L_{1}^{2}(T)^{2}}$

$$
+\left\|\frac{\left(\boldsymbol{u}^{n}-\boldsymbol{u}_{h}^{n}\right)-\left(\boldsymbol{u}^{n-1}-\boldsymbol{u}_{h}^{n-1}\right)}{\tau_{n}}+\boldsymbol{g r a d}\left(p^{n}-p_{h}^{n}\right)\right\|_{L_{1}^{2}(T)^{2}}^{2}+J_{T}^{n},
$$

where $J_{T}^{n}=\left\|\boldsymbol{f}^{n}-\Pi_{n h} \boldsymbol{f}^{n}\right\|_{L_{1}^{2}(T)^{2}}$.

Proof. Taking $\boldsymbol{v}=\left(\boldsymbol{u}_{h}^{n-1}-\Pi_{n h} \boldsymbol{u}_{h}^{n-1}\right) \chi_{T}$ in the first equation of (54), where $\chi_{T}$ is the charateristic function of $T$ and using the fact that

$p_{*}^{n}-p_{* h}^{n}=p^{n}-p_{h}^{n}-\mathcal{L}\left(p_{b}^{n}-i_{n h} p_{b}^{n}\right)$ we obtain

$\left\|\boldsymbol{u}_{h}^{n-1}-\Pi_{n h} \boldsymbol{u}_{h}^{n-1}\right\|_{L_{1}^{2}(T)^{2}}^{2}=\alpha \tau_{n}\left(\boldsymbol{u}^{n}-\boldsymbol{u}_{h}^{n}, \boldsymbol{u}_{h}^{n-1}-\Pi_{n h} \boldsymbol{u}_{h}^{n-1}\right)_{1, T}$

$$
\begin{gathered}
+\tau_{n}\left(\frac{\left(\boldsymbol{u}^{n}-\boldsymbol{u}_{h}^{n}\right)-\left(\boldsymbol{u}^{n-1}-\boldsymbol{u}_{h}^{n-1}\right)}{\tau_{n}}+\operatorname{grad}\left(p^{n}-p_{h}^{n}\right), \boldsymbol{u}_{h}^{n-1}-\Pi_{n h} \boldsymbol{u}_{h}^{n-1}\right)_{1, T} \\
-\tau_{n} \int_{T}\left(\boldsymbol{f}^{n}-\Pi_{n h} \boldsymbol{f}^{n}\right)\left(\boldsymbol{u}_{h}^{n-1}-\Pi_{n h} \boldsymbol{u}_{h}^{n-1}\right) r d r d z .
\end{gathered}
$$

Cauchy-Schwarz inequality yields

$$
\begin{gathered}
\left\|\boldsymbol{u}_{h}^{n-1}-\Pi_{n h} \boldsymbol{u}_{h}^{n-1}\right\|_{L_{1}^{2}(T)^{2}} \leq \alpha \tau_{n}\left\|\boldsymbol{u}^{n}-\boldsymbol{u}_{h}^{n}\right\|_{L_{1}^{2}(T)^{2}} \\
+\tau_{n}\left\|\frac{\left(\boldsymbol{u}^{n}-\mathbf{u}_{h}^{n}\right)-\left(\boldsymbol{u}^{n-1}-\boldsymbol{u}_{h}^{n-1}\right)}{\tau_{n}}+\operatorname{grad}\left(p^{n}-p_{h}^{n}\right)\right\|_{L_{1}^{2}(T)^{2}} \\
+\tau_{n}\left\|\boldsymbol{f}^{n}-\Pi_{n h} \boldsymbol{f}^{n}\right\|_{L_{1}^{2}(T)^{2}} .
\end{gathered}
$$

Finaly by multipling this inequality by $\frac{1}{\tau_{n}}$ and from the expression of the a posteriori indicator $\eta_{T}^{n}$, we get the desired estimate.

Proposition 10. For each $n, 1 \leq n \leq N$ and $T \in \mathcal{T}_{n h}$,

$$
\eta_{\partial T}^{n} \leq c\left\|\boldsymbol{u}^{n}-\boldsymbol{u}_{h}^{n}\right\|_{L_{1}^{2}\left(\omega_{T}\right)^{2}} .
$$

Proof. By means of a fixed lifting operator on the reference element $\widehat{T}$ and by using the affine transformation that maps $\widehat{T}$ onto $T$, we construct for each $e \in \mathcal{E}_{T}$ a lifting operator $\mathcal{L}_{e, T}$ such that for each polynomial $\varphi$ on $e$ vanishing on $\partial e, \mathcal{L}_{e, T} \varphi$ is a polynomial on $T$ vanishing on $\partial T \backslash e$ and equal to $\varphi$ on $e$. Let $b_{e}$ denote the bubble function on $e$, i.e., the product of the barycentric coordinates associated with the vertices of $e$. For each $e \in \mathcal{E}_{T}^{0}$, we denote by $T^{\prime}$ the other 
element of $\mathcal{T}_{n h}$ that contains $e$. In the second equation of (54), we take $q_{h}^{n}=0$ and $q=q_{e}^{n}$, with

$$
q_{e}^{n}=\left\{\begin{array}{lr}
\mathcal{L}_{e, T}\left(\left[\boldsymbol{u}_{h}^{n} \cdot \boldsymbol{n}_{e}\right]_{e} b_{e}\right) & \text { on } T \\
\mathcal{L}_{e, T^{\prime}}\left(\left[\boldsymbol{u}_{h}^{n} \cdot \boldsymbol{n}_{e}\right]_{e} b_{e}\right) & \text { on } T^{\prime} \\
0 & \text { elsewhere }
\end{array}\right.
$$

Then, we obtain

$$
b\left(\boldsymbol{u}^{n}-\boldsymbol{u}_{h}^{n}, q_{e}^{n}\right)=\left\langle g^{n}, q_{e}^{n}\right\rangle_{\Gamma_{u}}-\frac{1}{2} \sum_{T \in \mathcal{T}_{n h}}\left(\sum_{e \in \mathcal{E}_{n h}^{\Gamma u}} \int_{e}\left[\boldsymbol{u}_{h}^{n} \cdot \boldsymbol{n}_{e}\right]_{e}(\tau) q_{e}^{n}(\tau) d \tau\right) .
$$

On the other hand,

$q_{e}^{n}(\tau)=\mathcal{L}_{e, T}\left(\left[\boldsymbol{u}_{h}^{n} \cdot \boldsymbol{n}_{e}\right]_{e} b_{e}\right) \cdot \chi_{T}+\mathcal{L}_{e, T^{\prime}}\left(\left[\boldsymbol{u}_{h}^{n} \cdot \boldsymbol{n}_{e}\right]_{e} b_{e}\right) \cdot \chi_{T^{\prime}}=2\left[\boldsymbol{u}_{h}^{n} \cdot \boldsymbol{n}_{e}\right]_{e} b_{e}$.

Thus,

$$
\left\|\left[\boldsymbol{u}_{h}^{n} \cdot \boldsymbol{n}_{e}\right]_{e} b_{e}^{\frac{1}{2}}\right\|_{L_{1}^{2}(e)}^{2} \leq\left\|\boldsymbol{u}_{h}^{n}-\boldsymbol{u}^{n}\right\|_{L_{1}^{2}\left(T \cup T^{\prime}\right)^{2}}\left|q_{e}^{n}\right|_{H_{1}^{1}\left(T \cup T^{\prime}\right)} .
$$

Recall the following inverse inequality, for each constant $\lambda$, see [16, Lem. 3.3],

$$
\|\lambda\|_{L_{1}^{2}(e)} \leq c\left\|\lambda b_{e}^{\frac{1}{2}}\right\|_{L_{1}^{2}(e)} \quad \text { and } \quad\left|\mathcal{L}_{e, T}\left(\lambda b_{e}\right)\right|_{H_{1}^{1}(T)} \leq c h^{-\frac{1}{2}}\|\lambda\|_{L_{1}^{2}(e)} .
$$

The expression of $\eta_{\partial T}^{n}$ and the first inverse inequality, yields

$$
\eta_{\partial T}^{n} \leq c\left(\sum_{e \in \mathcal{E}_{n h}^{\Gamma u}} h_{e}^{\frac{1}{2}}\left\|\left[\boldsymbol{u}_{h}^{n} \cdot \boldsymbol{n}_{e}\right]_{e} b_{e}^{\frac{1}{2}}\right\|_{L_{1}^{2}(e)}\right) .
$$

Using estimate (58) we obtain

$$
\eta_{\partial T}^{n} \leq c\left(\sum_{e \in \mathcal{E}_{n h}^{\Gamma_{u}}} h_{e}^{\frac{1}{2}}\left\|\boldsymbol{u}_{h}^{n}-\boldsymbol{u}^{n}\right\|_{L_{1}^{2}\left(T \cup T^{\prime}\right)^{2}}^{\frac{1}{2}} \cdot\left|\mathcal{L}_{e, T}\left(\left[\boldsymbol{u}_{h}^{n} \cdot \boldsymbol{n}_{e}\right]_{e} b_{e}\right)\right|_{H_{1}^{1}\left(T \cup T^{\prime}\right)}^{\frac{1}{2}}\right) .
$$

By substituting the second inverse inequality into this last inequality we obtain

$$
\begin{aligned}
\eta_{\partial T}^{n} & \leq c\left(\sum_{e \in \mathcal{E}_{n h}^{\Gamma u}} h_{e}^{\frac{1}{4}}\left\|\boldsymbol{u}_{h}^{n}-\boldsymbol{u}^{n}\right\|_{L_{1}^{2}\left(T \cup T^{\prime}\right)^{2}}^{\frac{1}{2}} \cdot\left\|\left[\boldsymbol{u}_{h}^{n} \cdot \boldsymbol{n}_{e}\right]_{e}\right\|_{L_{1}^{2}(e)}^{\frac{1}{2}}\right) \\
& \leq c\left(\frac{1}{2} \sum_{e \in \mathcal{E}_{n h}^{\Gamma u}}\left\|\boldsymbol{u}_{h}-\boldsymbol{u}^{n}\right\|_{L_{1}^{2}\left(T \cup T^{\prime}\right)^{2}}+\frac{1}{2} \sum_{e \in \mathcal{E}_{n h}^{\Gamma u}} h_{e}^{\frac{1}{2}}\left\|\left[\boldsymbol{u}_{h}^{n} \cdot \boldsymbol{n}_{e}\right]_{e}\right\|_{L_{1}^{2}(e)}\right) .
\end{aligned}
$$

This gives gives the bound for the second indicator $\eta_{\partial T}^{n}$.

In both Propositions 9 and 10, the estimates are local in space and time, so that it can be thought that the indicators $\eta_{T}^{n}$ and $\eta_{\partial T}^{n}$ provide a good tool for adapting the mesh. 


\section{Some numerical experiments}

We present some numerical experiments realized with the code FreeFem++, see [11]. The domain $\breve{\Omega}$ is generated by the L-shaped meridian domain $\Omega$ defined by

$$
\Omega=] 0,1] \times] 0,0.5[\cup] 1,2[\times] 0,1[.
$$

We denote by $\Gamma_{p}$ the intersection of $\Gamma$ with the plan $z=0$ and $\Gamma_{u}$ is equal to $\Gamma \backslash \Gamma_{p}$.

We work with the data $g$ equal to one on $\Gamma_{u}=\Gamma \backslash \Gamma_{p}$ such that

$$
g(2, z)=g(r, 1)=g(1, z)=g(r, 0.5)=1 \quad \text { on } \Gamma_{u} .
$$

We take $p(0, z)=0$ on $\Gamma_{p}$, and the initial values of the velocity $\boldsymbol{u}$ in $\Omega$ at $t=0$ are such that

$$
u_{z}^{0}=0 \quad \text { and } \quad u_{r}^{0}=1
$$

The data $\mathbf{f}$ are such that

$$
f_{r}=1 \quad \text { and } \quad f_{z}=0 .
$$

Finally we take $\alpha$ equal to $0.25, d t=0.05$ and $T=1$.

Figure 1 presents, the curves of isovalues of the initial axial velocity $u_{z}^{0}$.

Figure 2 presents, the curves of isovalues of the initial axial velocity $u_{r}^{0}$.

Figure 3 presents, the curves of isovalues of the pressure at time $T=1$.

Figure 4 presents, the curves of isovalues and directions of the velocity at time $T=1$.

\section{Acknowledgements}

We thank Prof. Frédéric Hecht for his help in the numerical part of this work. His comments and suggestions were very useful to complete this research work.

\section{References}

[1] Y. Achdou, C. Bernardi, F. Coquel, A priori and a posteriori analysis of finite volume discretizations of Darcy's equations, Numer. Math. 96, (2003), pp. 17-42.

[2] M. Azaïez, F. Ben Belgacem, C. Bernardi, N. Chorfi, Spectral discretization of Darcy's equations with pressure dependent porosity, Applied Mathematics and Computation 217, (2010), pp. 1838-1856.

[3] Z. Belhachmi, C. Bernardi, S. Deparis, Weighted Clement operator and application to the finite element discretization of the axisymmetric Stokes problem, Numer. Math. 105 (2006), pp. 217-24\%.

[4] A. Bergam, C. Bernardi, Z. Mghazli, A posteriori analysis of the finite element discretization of some parabolic equations, Math. Comput. 74, (2005), pp. 1117-1138. 
[5] C. Bernardi, M. Dauge, Y. Maday, Polynomials in weighted Sobolev spaces: basics and trace liftings, Internal Report 92039, Laboratoire d'Analyse Numérique, Université Pierre et Marie Curie, Paris (1992).

[6] C. Bernardi, M. Dauge, Y. Maday and M. Azaïez, Spectral Methods for Axisymmetric Domains, Series in Applied Mathematics, vol. 3, GauthierVillars et North-Holland, 3 (1999).

[7] C. Bernardi, V. Girault, K. Rajagopal, Discretisation of an unsteady flow through a porous solid modeled by Darcy's equations, Mathematical Models and Methods in Applied Sciences, Vol. 18, No. 12 (2008), pp. 2087-2123.

[8] C. Bernardi, Y. Maday, F. Rapetti, Discrétisations variationelles de problèmes aux limites elliptiques, Collection Mathématiques et Applications $\mathbf{4 5}$, Springer-Verlag, (2004).

[9] V. Girault, P.-A. Raviart, Finite Element Approximation of the NavierStokes equations, Lecture Notes in Mathematics 749, Springer-Verlag, Berlin, (1979).

[10] V. Girault, P.-A. Raviart, Finite Element Methods for Navier-Stokes Equations. Theory and Algorithms, Springer-Verlag, Berlin, (1986).

[11] F. Hecht, New development in freefem++. J. Numer. Math. 20, (2012), pp. 251-265.

[12] D. Jerisson, C.E. Kenig, The inhomogeneous Dirichlet problem in Lipschitz domains, J. Funct. Anal. 130, (1995), pp. 161-219.

[13] C. Johnson, Y.-Y. Nie, V. Thomée, An a posteriori error estimate and adaptive timestep control for a backward Euler discretization of a parabolic problem, SIAM J. Number. Anal. 27, (1990), pp. 277-291.

[14] K.R. Rajagobal, On a hierarchy of approximate models for flows of incompressible fluids through porous solids, Math. Models and Methods in the Applied Sciences 17, (2007), pp. 215-252.

[15] L. R. Scott, S. Zhang, Finite element interpolation of non-smooth functions satisfying boundary conditions, Math. Comp. 54, (1900), pp. 483-493.

[16] R. Verfürth, A Review of A Posteriori Error Estimation and Adaptive Mesh-Refinement Techniques, Wiley, Teubner, (1996). 
Graph u

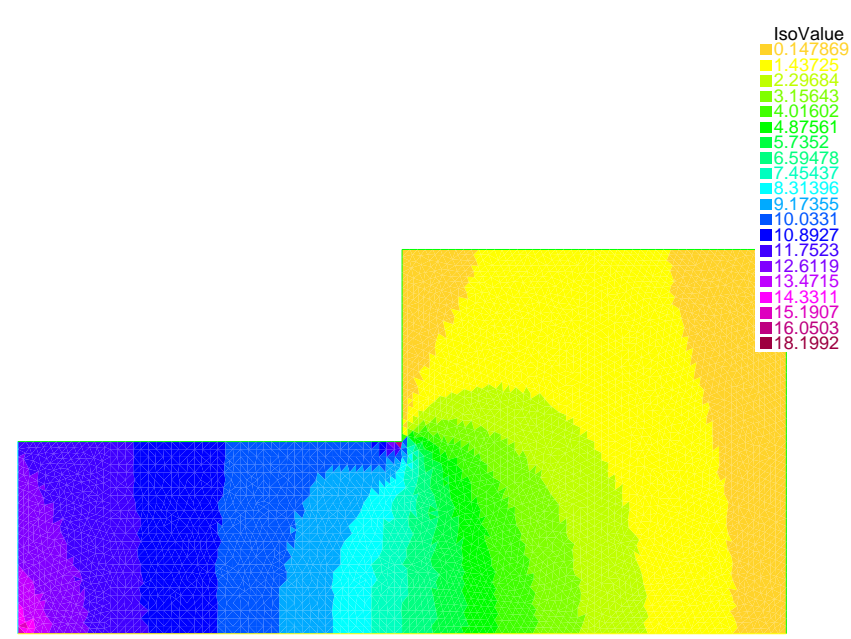

Figure 1: Isovalues of the initial axial velocity

Graph u

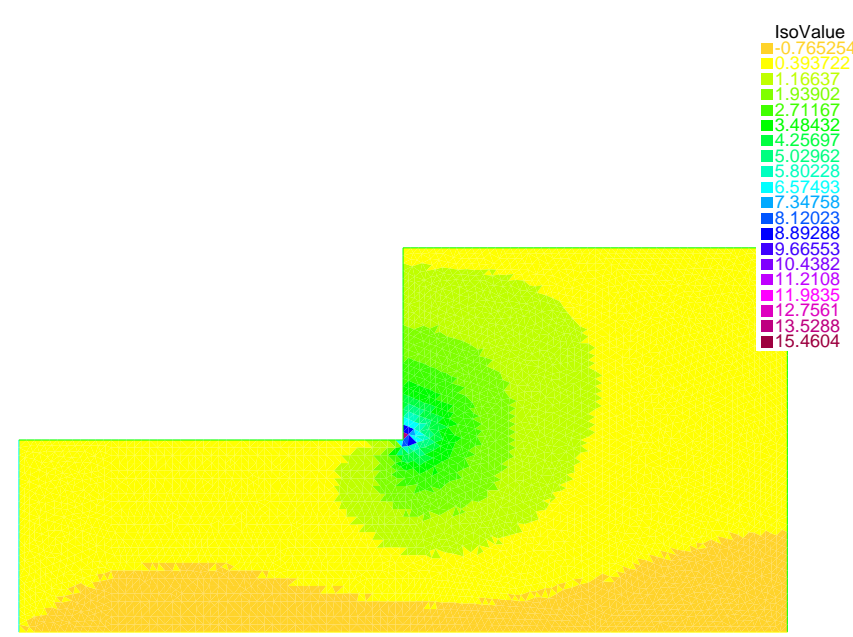

Figure 2: Isovalues of the initial radial velocity 


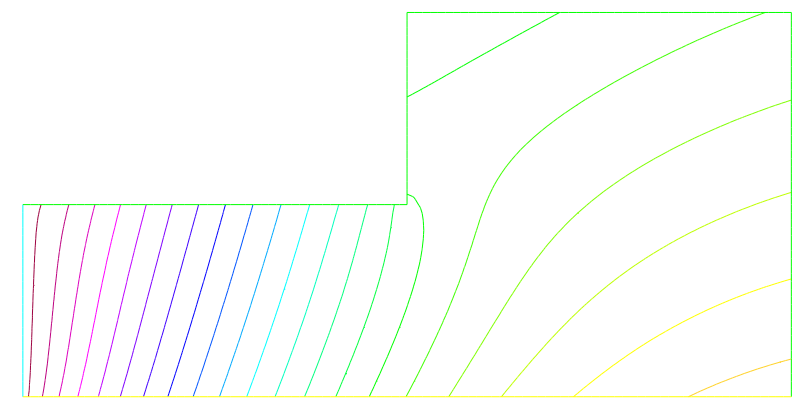

Figure 3: Isovalues of the pressure

graph u

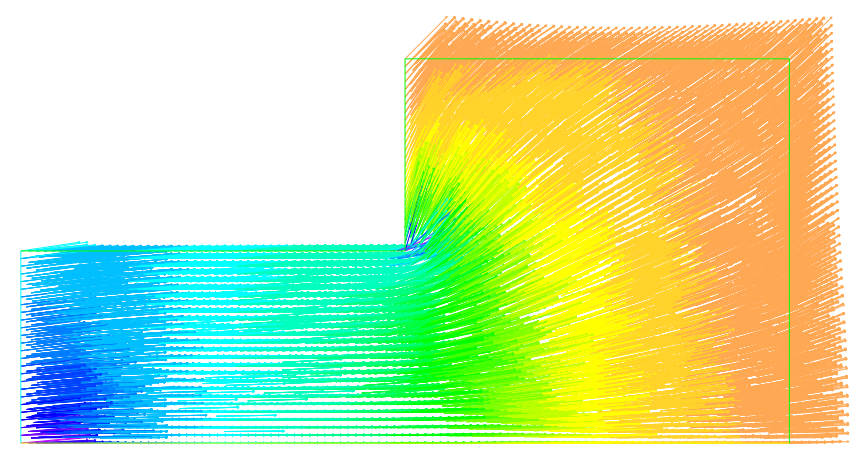

Figure 4: Isovalues and directions of the velocity 